\title{
Many-Body Approaches to Atoms and Molecules in External Magnetic Fields
}

\author{
MATTHEW D. JONES, ${ }^{1}$ GERARDO ORTIZ, ${ }^{1}$ DAVID M. CEPERLEY ${ }^{2}$ \\ ${ }^{1}$ Theoretical Division, Los Alamos National Laboratory, Los Alamos, New Mexico 87545 \\ ${ }^{2}$ Department of Physics, University of Illinois at Urbana-Champaign, 1110 West Green Street, \\ Urbana, Illinois 61801
}

Received 6 December 1996; revised 12 December 1996; revised 23 December 1996

\begin{abstract}
The proper treatment of many-body effects for fermions has long been a goal of theorists working in atomic and molecular physics. The computational demands of such a treatment, however, when coupled to the added difficulties imposed by the presence of external electromagnetic sources, have resulted in few studies of many-body effects in strong magnetic fields, i.e., in the field regime where perturbation theory is no longer applicable. In this article, we review the fundamental aspects of the problem and describe a variety of theoretical approaches for small atoms and molecules in strong fields, beginning with mean-field theory (Hartree-Fock) and progressing through variational and exact stochastic methods. (c) 1997 John Wiley \& Sons, Inc. Int J Quant Chem 64: 523-552, 1997
\end{abstract}

\section{Introduction}

The behavior of atoms and molecules in strong magnetic fields and its relation to the properties and stability of extended matter is a topic not limited only to theoretical interest. It is a subject of general interest due to their wide range of applications in different research areas, such as astrophysics and atomic, molecular, and condensed matter physics. With the discovery of pulsars and magnetized white dwarfs, the study of atomic [1] and molecular systems in strong magnetic fields has taken on a renewed importance. For many of these stars, the fields are strong enough to warrant

Correspondence to: G. Ortiz. a nonperturbative treatment. In particular, the surface of some neutron stars exhibits superintense fields strengths $\left(B \approx 10^{12} \mathrm{G}\right)$ which dramatically influence the structural and optical properties of matter. From the theoretical viewpoint, it is not clear whether a mean-field theory like Hartree-Fock is, in principle, able to capture the main physics of electron correlations, because of a nontrivial balance between coupled Lorentz and Coulomb forces. Moreover, most practical applications assume the adiabatic approximation [2] which amounts to retaining only the cylindrical symmetry imposed by the external field and which becomes asymptotically correct as $B \rightarrow \infty$. Consequently, to shed light on this issue, one has to resort to many-body methods that are better suited to deal with strongly correlated fermions. 
In this article, we are concerned with finite interacting spin- $1 / 2$ fermion systems in the presence of an external electromagnetic potential $A_{\mu}=$ $(\mathbf{A}, 0)(\mathbf{B}=\nabla \wedge \mathbf{A}$ represents a uniform field and A is the vector potential) characterized by the nonrelativistic Hamiltonian

$$
\hat{\mathbb{H}}=\sum_{i=1}^{\mathscr{N}} \frac{\left[\sigma_{i} \cdot \Pi_{i}\right]^{2}}{2 m_{i}}+V\left(\left\{\left|\mathbf{r}_{i}-\mathbf{r}_{j}\right|\right\}\right),
$$

where $\sigma_{i}^{k}, k=1,2,3$ denote the Pauli spin matrices and $\Pi_{i}=\mathbf{p}_{i}-\left(q_{i} / c\right) \mathbf{A}\left(\mathbf{r}_{i}\right)$ is the kinetic momentum. The first term in Eq. (1) is the Pauli kinetic energy and is the nonrelativistic approximation to the Dirac operator, while the second one represents the potential energy. Our system, in general, will be composed of two different kinds of particles ( $N$ electrons and $N_{+}$positive charges, such that $\mathscr{N}=N+N_{+}$) of mass, charge, vector position, and spin $m_{i}, q_{i}, \mathbf{r}_{i}=\left(x_{i}, y_{i}, z_{i}\right)$ and $s_{i}$, respectively. The many-particle wave functions $\Psi\left(\left\{\mathbf{r}_{i}\right\},\left\{s_{i}\right\}\right)$ and all its first derivatives belong to the Hilbert space of antisymmetric [with respect to identical particle $\left(\mathbf{r}_{i}, s_{i}\right)$ exchanges] square-integrable functions $\mathscr{H}=\mathscr{L}^{2}\left(\mathbb{R}^{3 r}\right) \otimes \mathbb{C}^{2 r}$.

Depending on the relative strength between Coulomb and Lorentz forces, we can characterize three different regimes: the low $\left(\beta \leq 10^{-3}\right)$, the intermediate or strong $\left(10^{-3} \leq \beta \leq 1\right)$, and superstrong $(\beta \gg 1)$ field regimes, where $\beta=e a_{0}^{2} B / 2 \hbar c$ $=B / B_{0} \quad\left(B_{0}=4.701 \times 10^{9} \mathrm{G}\right.$ and $a_{0}=\hbar^{2} / m e^{2}$, where $m$ and $e$ are the mass and charge of the electron). It is the strong and superstrong regimes which are relevant for white dwarf and neutron star physics, and it is this range of magnetic field strengths that will be our primary concern in this article. In the remainder of this section, we discuss the general properties and applications of the Hamiltonian $\hat{\mathbb{A}}$, review previous studies of these systems, and outline our general approach to obtaining solutions to Eq. (1).

\section{THE HAMILTONIAN AND ITS SYMMETRIES}

The stationary Schrödinger equation corresponding to $\hat{H}$ (i.e., $\hat{\uplus} \Psi=E \Psi$ ) is invariant under $U(1)$ transformations (charge conservation)

$$
\Psi \rightarrow \exp \left[i \sum_{i=1}^{\mathscr{N}} \frac{q_{i}}{\hbar c} \Lambda\left(\mathbf{r}_{i}\right)\right] \Psi,
$$

whenever the vector potential changes accordingly to

$$
\mathbf{A}\left(\mathbf{r}_{i}\right) \rightarrow \mathbf{A}\left(\mathbf{r}_{i}\right)+\nabla_{i} \Lambda\left(\mathbf{r}_{i}\right) .
$$

A finite magnetic translation by a vector $\mathbf{v}$ of particle $i$ is represented by the operator

$$
T(\mathbf{v})=\exp \left[i \mathbf{v} \cdot \frac{\mathbf{k}_{i}}{\hbar}\right],
$$

where $\mathbf{k}_{i}=\Pi_{i}+2\left(q_{i} / c\right) \mathbf{A}_{s}\left(\mathbf{r}_{i}\right)$ is the generator of infinitesimal magnetic translations and $\mathbf{A}_{s}\left(\mathbf{r}_{i}\right)=$ $B\left(-y_{i}, x_{i}, 0\right) / 2$ is the vector potential in the symmetric gauge. It turns out that the total "pseudomomentum" $\mathbf{K}_{0}=\sum_{i} \mathbf{k}_{i}$ is a constant of motion, i.e., $\left[\hat{\mathbb{H}}, \mathbf{K}_{0}\right]=0$. Notice that the components of $\mathbf{K}_{0}$ do not commute among themselves unless the system is neutral $\left(\sum_{i} q_{i}=0\right)$.

For an arbitrary gauge $\mathbf{A}$ such that $\mathbf{B}=B \hat{z}$, another symmetry of the above Hamiltonian is the $z$-component of the gauge-covariant operator $\tilde{\mathbf{L}}=$ $\sum_{i} \mathbf{r}_{i} \wedge\left[\Pi_{i}+\left(q_{i} / c\right) \mathbf{A}_{s}\right]$ [3]. The Cartesian components of this operator satisfy the algebra of angular momentum $\left[\tilde{L}_{\mu}, \tilde{L}_{\nu}\right]=i \hbar \epsilon_{\mu \nu \lambda} \tilde{L}_{\lambda}$ with the Casimir operator $\tilde{\mathbf{L}}^{2}\left(\left[\tilde{L}_{\mu}, \tilde{\mathbf{L}}^{2}\right]=0\right)$. Notice, that $\tilde{L}_{z}$ reduces, in the symmetric gauge, to $\hat{L}_{z}$, i.e., the $z$-component of the total angular momentum.

Whenever the mass difference between electrons and the positive charges is large, we will always assume the conventional Born-Oppenheimer adiabatic separation of electronic and nuclear motion, i.e., we will not include the effects of the non-Abelian and Mead-Berry connection in the slow variables induced by the fast electronic motion.* Besides, we are not concerned with enforcing the correct permutational symmetry on the total wave function with respect to identical nuclei exchanges and consider only the electron dynamics which, in turn, depends parametrically on the nuclear space coordinates.

\section{THE SOLID-STATE CONNECTION}

The study of strongly magnetized atoms was first treated in a solid-state context. In semiconductors, the absorption of photons can promote electrons from the valence to the conduction band, forming an electron-hole pair. The absorption spectrum of these excitons, when subjected to a magnetic field, display Zeeman effects. These effects can be modeled by considering a hydrogen

* This fact can be relevant in the presence of conical intersections of the adiabatic potential energy hypersurfaces, as in $\mathrm{H}_{3}$. See, for instance, [4]. 
atom with an electron whose mass $m$ has been replaced by an effective mass $m^{*}$ and a proton whose mass is replaced by an effective mass $m_{h}$. The usual Coulomb potential is screened by an effective dielectric constant, $\epsilon$. In the presence of a constant magnetic field (taken without loss of generality to be in the $z$ direction), the Hamiltonian for this system is given by

$$
\begin{aligned}
-\nabla^{2}-\frac{2 \mu}{m^{*} r}+2 \beta^{*}\left(\frac{m_{h}-m^{*}}{m_{h}+m^{*}}\right) & \hat{L}_{z} \\
& +\beta^{* 2}\left(x^{2}+y^{2}\right),
\end{aligned}
$$

where the energy scale is now in scaled rydbergs, $\mathrm{Ry}^{*}=\left(m^{*} / m \epsilon^{2}\right) \mathrm{Ry}$, lengths are relative, $\beta^{*}=$ $\beta\left(m \epsilon / m^{*}\right)^{2}$, and $\mu$ is the reduced mass. The constant spin contribution has been neglected. For $m_{h} \gg m^{*}$, the above Hamiltonian reduces to that of magnetized hydrogen and was first derived and discussed by Elliott and Louden [5]. In certain semiconductors, the dielectric constant is large enough and the effective mass small enough to have very large effective field strengths in even a moderate laboratory field. An extreme example is that of indium antimonide ( $\mathrm{InSb}$ ), where $m^{*}=$ $0.013 m$ and $\epsilon=16$, which results in $\beta^{*}=1.5 \times$ $10^{6} \beta$. Thus, a $1 \mathrm{~T}=10^{4} \mathrm{G}$ laboratory field results in an effective field of $\beta^{*} \simeq 3.2$, which corresponds to an isolated hydrogen atom in a field of more than $10^{10} \mathrm{G}$. To a good approximation, such excitons can be studied (with appropriate scaling) by considering the case of an isolated atom. The atomic calculations are also relevant for quantum dots [6].

\section{APPLICATIONS IN ASTROPHYSICS}

Some dense compact stellar remnants possess very large magnetic fields, from magnetic white dwarfs to neutron stars. The first direct measurement of the magnetic field of a neutron star by Trümper et al. [7] observed a cyclotron emission line in the Hercules X-1 pulsar corresponding to a field strength of approximately $10^{12} \mathrm{G}$. This discovery intensified research into atomic electronic structure within the adiabatic approximation, which we review below.

Angel [8] first proposed that magnetically altered "stationary" spectral lines could account for unidentifiable spectral features of some magnetized white dwarfs. Lines are considered stationary if they are effectively constant over a range of magnetic field strength. The natural variation of magnetic field over the surface of a star (a factor of two in even a simple dipole model) would smear any spectral lines that were rapidly varying as a function of field strength. Angel's suggestion stimulated much interest in the accurate calculation of atomic states, which culminated in an accurate determination of the hydrogen spectrum [9] that has been successfully applied to many previously unexplained spectra. Several stars, however, have spectral properties that remain unexplained. Neutral helium has been one suggestion $[10,11]$ to account for the unexplained spectra.

\section{PREVIOUS WORK ON MAGNETIZED ATOMS AND MOLECULES}

With the exception of hydrogen, our theoretical knowledge of the behavior of these systems has not kept pace with the experimental work. It has been one of the the primary goals of our work to help fill that gap. In a regime where Coulomb and magnetic effects are of nearly equal importance, and neither can be treated as a perturbation, lie many interesting applications. Unfortunately, this regime is also very difficult to solve, since the combination of the cylindrical symmetry of the magnetic field and the spherical symmetry of the Coulomb potential prevents the Schrödinger equation from being separable or integrable. Previous approaches to this problem have been concentrated on the two limits: very weak magnetic fields (where the magnetic field may be treated as a perturbation [12]) and very strong magnetic fields (where cylindrical symmetry is imposed on the wave function in the so-called adiabatic approximation [2]). Several recent works, however, have attempted to bridge this gap and deal with the intermediate (or strong field) regime. For atomic systems, we will work in dimensionless atomic units through use of the parameter $\beta_{Z}=B / B_{0} Z^{2}$ $=\beta / Z^{2}$.

Rosner et al. [9] used the numerical HartreeFock (HF) method to calculate many states of the hydrogen atom $(Z=1)$ in fields up to $\beta_{Z} \leq 10^{3}$. In these computations, the wave function is expanded in terms of either spherical harmonics or Landau-like orbitals (adiabatic basis set) depending on the field strength, and the resulting Hartree-Fock equations are then integrated numerically. This set of computed energies and oscillator strengths has been used to identify many special features of compact stellar remnants and is often used as a benchmark for other methods [1]. 
For systems involving more than one electron, efforts have largely been concentrated on the high-field (adiabatic) regime, where the magnetic forces dominate and the wave function is assumed to have cylindrical symmetry. Mueller et al. [13] used cylindrical trial wave functions to compute variational upper bounds for the lowest-energy states of $\mathrm{H}^{-}, \mathrm{He}$ and $\mathrm{Li}^{+}$in the adiabatic approximation for fields in excess of $10^{10} \mathrm{G}$. Vincke and Baye [14] also obtained variational estimates for $\mathrm{H}^{-}$and $\mathrm{He}$ for magnetic fields in excess of $10^{10} \mathrm{G}$. Again, in the adiabatic limit, with fields larger than $10^{11}$ G, Miller and Neuhauser [15] and Neuhauser et al. [16] used HF methods on small atoms and molecular chains. Lai et al. [17] also used HF methods to treat hydrogen molecular chains in the adiabatic limit. Work at such strong magnetic fields, while not complete, is made somewhat easier by the dominance of the magnetic field in this regime. The situation at intermediate fields is more complex.

At intermediate field strengths, the nearly equal importance of Coulomb and magnetic effects has made progress very difficult, and, thus far, only two-electron problems have been attempted. This range of field strengths is plagued by the fact that neither the spherical symmetry of the Coulombic potential nor the cylindrical symmetry of the constant magnetic field may be assumed to dominate. Thorough HF calculations using the same method as Rosner et al.'s hydrogenic calculations [9] have been performed for heliumlike atoms [18], but these results, as we shall see, have difficulty in the region where the cylindrical and spherical expansions meet. Less complete variational calculations were performed for $\mathrm{H}^{-}$by Henry et al. [19] ( $\beta_{Z}<$ 0.2 ), and for He by Surmelian et al. [20] for $\beta_{Z}<20$. Both sets of variational calculations used a trial wave function which was a sum of slater orbitals. Larsen [21] also performed variational calculations on several low-lying excited states of $\mathrm{H}^{-}$and $\mathrm{He}$ for various fields of $\beta_{Z} \leq 5$, paying particular attention to the binding energy of $\mathrm{H}^{-}$.

\section{PRESENT WORK}

We studied multielectron atoms in this intermediate regime, using a basis-set HF approach that is flexible enough to balance the competing symmetries between the Coulomb and magnetic interac- tions. This work was the precursor of a quantum Monte Carlo (QMC) study of electron correlation at high magnetic fields in atomic systems, which is the subject of the subsections Introduction and Released-Phase QMC under the Stochastic Methods in Strong Magnetic Fields section. The QMC method is greatly aided by high-quality trial wave functions-hence, our immediate need for the calculations presented here. At the same time, the methods and tables presented in [3, 22-24] and reviewed here may be of use to astrophysicists analyzing unexplained spectra from magnetic white dwarf stars, e.g., GD229, for which helium has been suggested as a possible explanation for the pronounced yet nonhydrogenic spectral features [10]. We will reconsider the spectrum of neutral helium after discussing our HF method and its applications. Our QMC studies of magnetized systems will be introduced in the fourth section as a way to overcome to the limitations of the mean-field method. Using our Fixed-phase (FPQMC) and released-phase (RPQMC) quantum Monte Carlo methods will enable us to compute highly accurate, statistically "exact," energies of magnetized systems.

We also considered the behavior of the lowest excitations of the hydrogen molecule in the superstrong regime [3]. Our results [3], which we review below in the subsection The $\mathrm{H}_{2}$ Molecule, clearly indicate that $\mathrm{H}_{2}$ in superstrong magnetic fields tends to form tightly bonded molecules and not a weakly interacting Bose gas [25-27] as has been recently suggested. Our method of choice for this system has again been QMC; we will for the first time present results for the RPQMC energies of selected states, which will quantitatively demonstrate the essential weaknesses of the mean-field approach.

\section{Hartree-Fock Methods for Magnetized Atoms}

\section{THE HARTREE-FOCK EQUATIONS}

We begin our study of magnetized atoms with a mean-field approach. Later, we will consider a more rigorous treatment that includes the effects of electron-electron correlation. The Hamiltonian in Hartree atomic units (1 Hartree $=\hbar^{2} / m a_{0}^{2}$ ) for an $\mathrm{N}$-electron atom in constant magnetic field is 
given by

$$
\begin{aligned}
\hat{\mathscr{H}}=\sum_{i=1}^{N}\left[-\frac{\nabla_{i}^{2}}{2}\right. & \left.-\frac{Z}{r_{i}}+\frac{\beta^{2}}{2}\left(x_{i}^{2}+y_{i}^{2}\right)\right] \\
& +\beta\left(\hat{L}_{z}+2 \hat{S}_{z}\right)+\sum_{1 \leq i<j \leq N} \frac{1}{r_{i j}},
\end{aligned}
$$

where $\hat{L}_{z}=\sum_{i=1}^{N} \hat{\ell}_{i z}, \hat{S}_{z}=\sum_{i=1}^{N} \hat{s}_{i z}$, and lengths are in units of the bohr radius $a_{0}$. We chose the magnetic field to be parallel to the $z$-axis and the symmetric gauge. In the absence of external fields, the eigenvalues of $\hat{L}^{2}, \hat{L}_{z}, \hat{S}^{2}, \hat{S}_{z}$, and parity, $\hat{\Pi}$ are good quantum numbers. When the magnetic field is turned on, the rotational invariance is broken and the only conserved quantum numbers are the eigenvalues of $\hat{L}_{z}, \hat{S}^{2}, \hat{S}_{z}$ (i.e., $\hat{L}_{z} \Psi=M \Psi, \hat{S}^{2} \Psi=$ $S(S+1) \Psi$ and $\left.\hat{S}_{z} \Psi=M_{S} \Psi\right)$, and $\hat{\Pi}$ (alternatively, we will use the $z$-parity, $\hat{\pi}_{z}$ ). With a different choice of gauge, $\hat{L}_{z}$ no longer commutes with the Hamiltonian; instead, one must use the gauge-covariant form (see subsection The Hamiltonian and Its Symmetries). The diamagnetic term $\left[\sim \beta^{2}\left(x^{2}+\right.\right.$ $\left.y^{2}\right)$ ] in the Hamiltonian couples states that differ by two in $l$. In large fields, e.g., our labeling an electronic state as $1 \mathrm{~s}$ denotes the first state of even parity $s+d+g+\cdots$, which would be the familiar $1 s$ state at zero field.

We take our total $N$ electron wavefunction to be composed of a single Slater determinant of single-particle spin orbitals,

$$
|\Psi\rangle=\left|\psi_{1} \ldots \psi_{N}\right\rangle,
$$

where $\psi_{a}(\mathbf{x})=\alpha(s) \otimes \phi_{a}(\mathbf{r}), \alpha(s)$ is a spin function, $\phi_{a}(\mathbf{r})$ a spatial orbital, and $\mathbf{x}=(\mathbf{r}, s)$. Minimization of the total energy,

$$
E\left[\left\{\psi_{a}\right\}\right]=\frac{\langle\Psi|\hat{\mathbb{H}}| \Psi\rangle}{\langle\Psi \mid \Psi\rangle},
$$

with respect to the spin orbitals then leads to the HF equations,

$$
F \psi_{a}=\epsilon_{a} \psi_{a}^{\prime}
$$

where $F$ is the single-particle Fock operator,

$$
F=h(\mathbf{r})+\sum_{b}\left(\mathscr{J}_{b}-\mathscr{K}_{b}\right),
$$

and

$$
\begin{aligned}
h(\mathbf{r})= & -\frac{1}{2} \nabla^{2}-\frac{Z}{r}+\frac{\beta^{2}}{2}\left(x^{2}+y^{2}\right) \\
& +\beta\left(\hat{\ell}_{z}+2 \hat{s}_{z}\right), \\
\mathscr{J}_{b} \psi_{a}= & {\left[\int d \mathbf{x}^{\prime}\left|\mathbf{r}-\mathbf{r}^{\prime}\right|^{-1} \psi_{b}^{*}\left(\mathbf{x}^{\prime}\right) \psi_{b}\left(\mathbf{x}^{\prime}\right)\right] \psi_{a}(\mathbf{x}), } \\
\mathscr{K}_{b} \psi_{a}= & {\left[\int d \mathbf{x}^{\prime}\left|\mathbf{r}-\mathbf{r}^{\prime}\right|^{-1} \psi_{b}^{*}\left(\mathbf{x}^{\prime}\right) \psi_{a}\left(\mathbf{x}^{\prime}\right)\right] \psi_{b}(\mathbf{x}) . }
\end{aligned}
$$

Note that we are still considering the integrals over the spin degrees of freedom for the direct, $\mathscr{J}$, and exchange, $\mathscr{K}$, integrals. We should also note, however, that we have made an approximation by choosing such a simple form for $|\Psi\rangle$. Since the wave function consists only of products of onebody orbitals, we have neglected many-body correlation effects. This assumption results in a set of equations in which each electron is treated as if it were moving in the average potential created by the other electrons (the direct integral) with an additional term arising from the quantum statistics (the nonlocal exchange integral). In subsequent sections, we will explore methods for moving beyond this mean-field approach.

\section{DIFFICULTIES OF THE MAGNETIZED COULOMB PROBLEM}

Considering that even magnetized hydrogen resisted an accurate HF solution for so long, we have to wonder what makes the magnetic field so difficult to handle. Let us consider, for simplicity, the one-electron atom. We may expand the spatial part of the wave function in terms of spherical harmonics, $Y_{l m}(\theta, \phi)$,

$$
\Psi(\mathbf{r})=\sum_{l} f_{l}(r) Y_{l m}(\theta, \phi) .
$$

The HF equation then results in a simple one-dimensional differential equation for the $\left\{f_{l}(r)\right\}$ functions (in units of Rydbergs),

$$
\begin{array}{r}
-E f_{l}(r)-d^{2} f_{l} / d r^{2}+\sum_{l^{\prime}} V_{l l^{\prime}} f_{l^{\prime}}(r)=0, \\
V_{l l^{\prime}}=\left\langle Y_{l m}\right| l(l+1) / r^{2}-1 / r+\beta\left(\hat{\ell}_{z}+2 \hat{s}_{z}\right) \\
+\left(\beta^{2} / 2\right) r^{2} \sin ^{2} \theta\left|Y_{l^{\prime} m}\right\rangle .
\end{array}
$$

where $\hat{s}_{z}$ is the electron spin projection in the direction of the applied field and the sum over 
$l^{\prime} \geq l$ is over all odd integers for odd $l$ and all even integers for even $l$. This effective potential is the principle difficulty to be overcome in performing HF calculations in strong magnetic fields, as many terms must be included in the sum as the field strength increases. This coupling between different $l$-values arises directly from the diamagnetic term in the Hamiltonian, making the problem inherently multiconfigurational. In practice, the above equations are solved iteratively, with $f_{0}$ used in computing $f_{1}, f_{1}$ in $f_{2}$, and so on until a self-consistent solution is reached. A different expansion scheme may be used, e.g., using an expansion in Landau orbitals, $\Phi_{n m}(\rho, \phi)$,

$$
\Psi(\mathbf{r})=\sum_{n} g_{n}(z) \Phi_{n m}(\rho, \phi) .
$$

In this case, the resulting HF equation is quite similar to Eq. (13):

$$
\begin{gathered}
-E g_{n}-d^{2} g_{n} / d z^{2}+\sum_{n^{\prime}} V_{n n^{\prime}} g_{n^{\prime}}=0 \\
V_{n n^{\prime}}=\left\langle\Phi_{n m}\left|\frac{-2}{r}\right| \Phi_{n^{\prime} m}\right\rangle .
\end{gathered}
$$

The adiabatic limit corresponds to ignoring the $n^{\prime} \neq n$ terms in Eq. (16), a questionable approximation except in the case of asymptotically large field strengths. This approach also suffers from the fact that a cylindrical separation is imposed on the overall wave function, which again is valid only the limit of very large fields. The most accurate calculations to date for hydrogen [18] have used a combination of these two approaches, computing many excited states in fields of $10^{-4} \leq \beta_{Z} \leq 10^{3}$ by numerical integration of the differential equations Eqs. (13) and (16). A similar approach can be used for multielectron systems, but thus far has only been attempted on two-electron systems [9] for the whole range of magnetic field strengths. Our method for attacking the HF equations, presented in the following section, will readily handle multielectron atoms and will be compared to the calculations performed on helium using the above method [9].

The necessity of many higher-order terms in the expansion of the wave function can be understood by carefully considering the terms in the Hamiltonian. The atom will seek to minimize its diamagnetic energy by shrinking in the direction transverse to the field. As the atom shrinks (and the field grows larger), the departure from spherical symmetry increases, and the high-order spherical harmonics (or Landau orbitals) are used to describe the rapidly changing electronic orbitals near the nucleus. As an example, consider Figure 1, which shows our HF results (discussed below) for the first three excited states of helium that are spherically symmetric at zero field. Note that, at zero field, the density behaves much as expected, with the most spatially extended state corresponding to the highest excitation. With an applied field of $\beta_{Z}=0.1$, however, we see that the atom is quite strongly pinched in the transverse $(x)$ direction, with all three excitations having negligible electron density more than 5 bohr radii away from the nucleus. The electron density in the longitudinal direction is also confined (lower plot) by the magnetic field, but much less so than the transverse direction (inset). This behavior makes a careful consideration of the number of angular momentum components (number of configurations) necessary.

\section{METHOD OF SOLUTION}

We used two different approaches to this HF problem, which differ in their assumptions about the initial Slater determinant used in the minimization of the variational energy. The first is spin-restricted Hartree-Fock (RHF), in which spin orbitals are pure space-spin products to be occupied in pairs, with a common spatial orbital factor. The second method is spin-unrestricted (UHF), in which the orbitals corresponding to different spins are allowed to differ. Although UHF generally obtains a better variational energy than does RHF for open-shell configurations, UHF wave functions, unlike their RHF counterparts, are not eigenstates of $\hat{S}^{2}$. It is more physical to consider states that are eigenfunctions of the total spin. For simplicity, however, we occasionally relax this constraint. We briefly outline the approach that we have taken for using UHF and RHF and further elaborate on the advantages and disadvantages of these formulations.

\section{Unrestricted Hartree-Fock}

The UHF method is formally much simpler than that of RHF. We begin with a single Slater determinant consisting of single-particle spin orbitals in which the spatial orbitals corresponding to unlike 


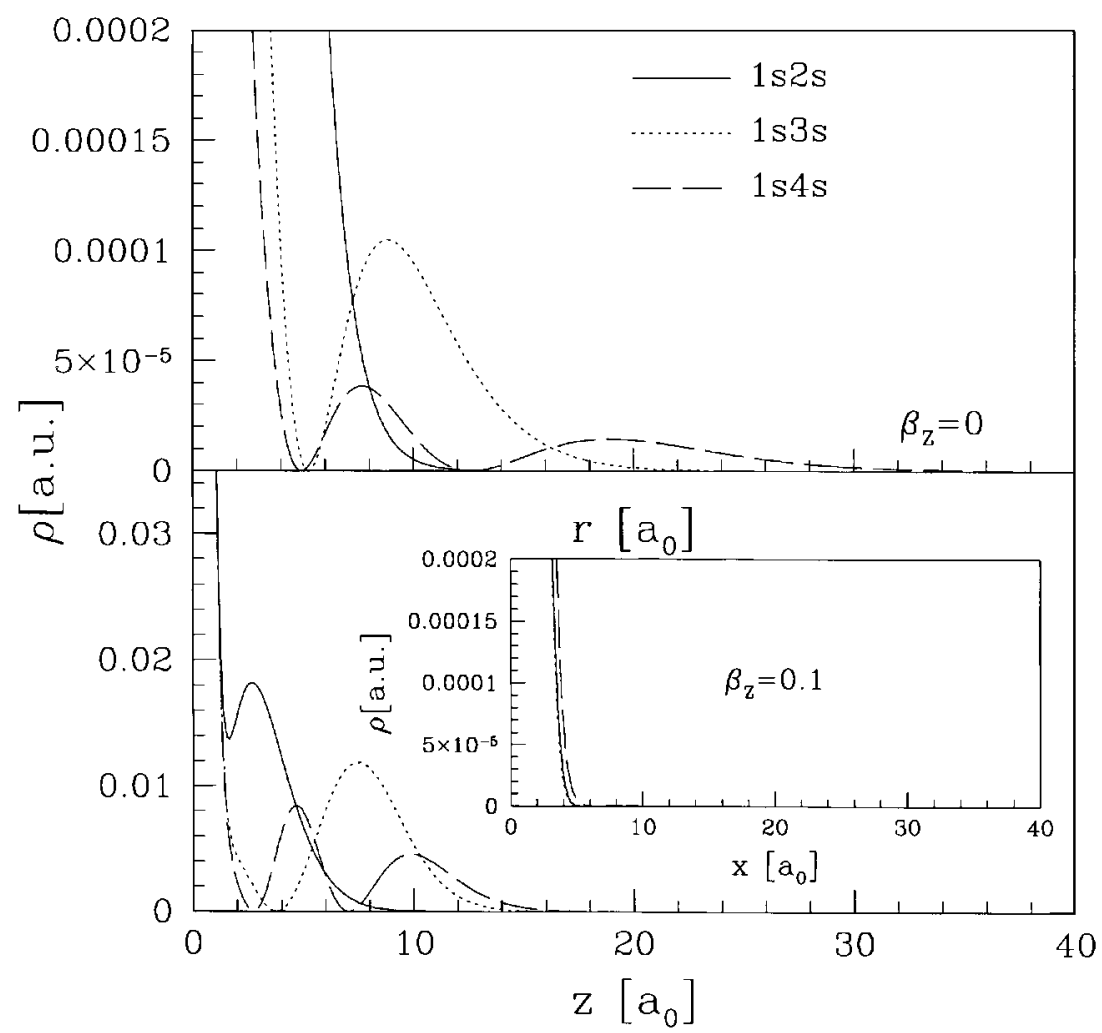

FIGURE 1. The HF electron density in the longitudinal $(z)$ and transverse $(x)$ directions for the first three excited states of helium that are spherically symmetric at zero field (top plot). The bottom plot shows the resulting anisotropy in the electron density when a field of $\beta_{Z}=0.1$ is applied.

spins are allowed, to differ:

$$
|\Psi\rangle=\left|\phi_{1}^{\alpha} \alpha\left(s_{1}\right) \phi_{1}^{\beta} \beta\left(s_{1}\right) \ldots \phi_{N}^{\alpha} \alpha\left(s_{N}\right) \phi_{N}^{\beta} \beta\left(s_{N}\right)\right\rangle .
$$

Minimization of the variational energy of this wave function then results in the UHF equations (eigenvalue equations for each spin type),

$$
\begin{aligned}
& \hat{f}^{\alpha}(\mathbf{r}) \phi_{a}^{\alpha}(\mathbf{r})=\boldsymbol{\epsilon}_{a}^{\alpha} \phi_{a}^{\alpha}(\mathbf{r}), \\
& \hat{f}^{\beta}(\mathbf{r}) \phi_{a}^{\beta}(\mathbf{r})=\boldsymbol{\epsilon}_{a}^{\beta} \phi_{a}^{\beta}(\mathbf{r}),
\end{aligned}
$$

where $\hat{f}(\mathbf{r})$ is the single-body operator,

$$
\begin{aligned}
& \hat{f}^{\alpha}\left(\mathbf{r}_{1}\right) \phi_{a}^{\alpha}\left(\mathbf{r}_{1}\right)=h\left(\mathbf{r}_{1}\right) \phi_{a}^{\alpha}\left(\mathbf{r}_{1}\right) \\
& \quad+\sum_{b=1}^{N_{\alpha}}\left[J_{b}^{\alpha}-K_{b}^{\alpha}\right] \phi_{a}^{\alpha}\left(\mathbf{r}_{1}\right)+\sum_{b=1}^{N_{\beta}} J_{b}^{\beta} \phi_{a}^{\alpha}\left(\mathbf{r}_{1}\right),
\end{aligned}
$$

and $\alpha$ and $\beta$ denote the two possible spin species $(s=1 / 2)$. To obtain the UHF solutions, one introduces a basis and simultaneously solves the coupled equations for the two-spin species. This method, due to the added freedom of the different spatial orbitals for unlike spins, can often have a lower variational energy than that of restricted HF, at the expense of the wave function no longer being an eigenfunction of $\hat{S}^{2}$. UHF is also considerably easier to implement, as it does not require a separate treatment of atoms with closed and open electronic shells.

\section{Restricted Hartree-Fock}

In the RHF approach, there are two important cases to be considered: closed electronic shells and open shells. The closed-shell RHF technique, developed as an algebraic problem with basis-set expansions by Roothaan [28], is one of the simplest to implement, but suffers from limited applicability (due to the relative prevalence of open-shell systems). For closed electronic shells, one solves

$$
\hat{f} \phi_{a}(\mathbf{r})=h(\mathbf{r}) \phi_{a}(\mathbf{r})+\sum_{b=1}^{N / 2}\left[2 J_{b}-K_{b}\right] \phi_{a}(\mathbf{r}),
$$


where we performed the integral over spin for $\mathscr{J}$ and $\mathscr{K}$, denoting the resulting direct and exchange spatial integrals $J$ and $K$. After introducing a basis for the orbitals, one solves the resulting matrix equation for the basis-set coefficients $\left\{c_{a \mu}\right\}$. This simple sum over direct and exchange contributions when each orbital is doubly occupied becomes more complex in the open-shell case. For open-shell RHF, many different approaches have been formulated [29]. We have settled on an approach based on that of Guest and Saunders [30] which has the advantage of allowing for easier convergence to excited states. This method formulates a generalized matrix such that convergence is obtained with the matrix elements between occupied and unoccupied orbitals vanish. If $T_{1}, T_{2}$, and $T_{3}$ denote matrices whose columns are eigenvectors representing closed, open, and empty orbitals, respectively, then let

$\bar{H}=\left(\begin{array}{ccc}T_{1}^{\dagger} H_{d} T_{1} & \lambda_{12} T_{1}^{\dagger} H_{3} T_{2} & \lambda_{13} T_{1}^{\dagger} H_{1} T_{3} \\ \lambda_{12} T_{2}^{\dagger} H_{3} T_{1} & T_{2}^{\dagger} H_{p} T_{2}+\delta_{1} I & \lambda_{23} T_{2}^{\dagger} H_{2} T_{3} \\ \lambda_{13} T_{3}^{\dagger} H_{1} T_{1} & \lambda_{23} T_{3}^{\dagger} H_{2} T_{2} & T_{3}^{\dagger} H_{v} T_{3}+\left(\delta_{1}+\delta_{2}\right) I\end{array}\right)$,

where $I$ is the identity matrix; $\lambda_{i j}, \delta_{1}$, and $\delta_{2}$ are arbitrary parameters to improve convergence, and $H_{n}(n=1,2,3, d, p, v)$ is a Hermitian matrix whose form depends on the electronic configuration [30]. To obtain self-consistent solutions for the orbitals, one begins with a trial set of solutions, then repeats the process of computing and diagonalizing $\bar{H}$ until convergence is achieved. This method for converging open-shell RHF solutions is very flexible due to the rather large number of free parameters in Eq. (22) and the explicit decoupling of occupied and unoccupied orbitals.

\section{UHF or RHF?}

As we have discussed, with UHF, one does not need elaborate procedures to accommodate different open-shell situations; all electronic configurations are treated within the same formalism as given above in Eqs. (19). Primarily for this ease of use, we used UHF for all of the ground-state calculations performed in this work and RHF for the higher-excited states of neutral helium. The reason behind this choice of method is stability; we found the RHF method much more stable than UHF for highly excited states. The possible mixing of occupied and unoccupied states in UHF solu- tions often results in convergence to a state of lower energy. In the discussion of results presented below, we will be careful to label which HF method was used.

\section{APPLICATION OF A BASIS}

Rather than integrate the HF equations numerically on a radial grid, we choose to expand each electronic orbital in a basis set of dimension $N_{b},\left\{\chi_{\mu}(\mathbf{r})\right\}$, of our choosing:

$$
\phi_{a}(\mathbf{r})=\sum_{\mu=1}^{N_{b}} c_{a \mu} \chi_{\mu}(\mathbf{r}) .
$$

This expansion reduces the problem to an algebraic one, upon which well-established and robust solution methods can be brought to bear. One is also free to choose the dominant symmetry for a given magnetic field regime. In the adiabatic limit, we can use cylindrical basis functions (Landau-like orbitals), while at lower fields strengths it is more advantageous to use basis elements with spherical symmetry. For the intermediate range of magnetic field strength considered here, we chose to use either Slater-type orbitals (STO) or Gaussian-type orbitals (GTO) as our basis elements. Both STO and GTO have the same functional form,

$$
\chi_{\mu}(\mathbf{r})=R_{\mu}(r) Y_{l_{\mu} m_{\mu}}(\theta, \phi),
$$

where

$$
\begin{aligned}
R_{\mu}^{\mathrm{STO}}(r) & =N_{\mu}^{\mathrm{STO}} r^{n_{\mu}-1} e^{-a_{\mu} r}, \\
R_{\mu}^{\mathrm{GTO}}(r) & =N_{\mu}^{\mathrm{GTO}} r^{n_{\mu}-1} e^{-a_{\mu} r^{2}},
\end{aligned}
$$

with the normalization constants

$$
\begin{gathered}
N_{\mu}^{\mathrm{STO}}=\left[\left(2 a_{\mu}\right)^{\left(2 n_{\mu}+1\right)} /\left(2 n_{\mu}\right) !\right]^{1 / 2}, \\
N_{\mu}^{\mathrm{GTO}}=\left(2 a_{\mu} / \pi\right)^{1 / 4}\left[8 a_{\mu} /\left(2 n_{\mu}-1\right) ! !\right]^{1 / 2} .
\end{gathered}
$$

Note that this basis-set formulation has a distinct advantage over the direct radial-grid integration method in this particular application of atoms in magnetic fields. With direct integration of the HF equations [Eq. (9)], one must make an assumption about the symmetry of the wave function to reduce the partial differential equation to a more manageable (one-dimensional) ordinary one. At intermediate field strengths, neither cylindrical nor spherical symmetry dominates, making such 
an assumption hazardous. With the basis-set formalism, one can simply add more basis elements of different symmetry in a systemic way. In practice, we optimize the exponents, $\left\{a_{\mu}\right\}$, of the basis functions using a conjugate gradient approach, then attempt to saturate any remaining freedom in the basis with additional basis elements. One should also note that the STO basis is complete for any field strength, even if many terms of higher order in $l$ must be included. This advantage is not shared by the adiabatic basis set, in which the wave function is assumed to posses cylindrical symmetry. The individual matrix elements for the STO basis, even in magnetic fields, are straightforward [28], with the exception of the electron-electron interaction. The complete set of matrix elements and our method for efficiently computing the electron-electron integrals for both STO and GTO basis sets are presented in detail in [23].

\section{Optimal Basis Sets for Magnetized Atoms}

We have already noted that, for intermediate field strengths, the adiabatic basis set will not be the optimal choice. We also considered two different basis sets, Slater- and Gaussian-type orbitals. Which of these two basis sets is the best for atomic calculations in strong magnetic fields? First, we must decide on a criteron for which we can judge the relative merits of each basis set. There are two considerations: ease of calculation and the required number of basis elements to achieve a certain level of accuracy. The GTO functions are considerably easier to use in evaluating the necessary integrals. Indeed, GTOs are the only basis set generally used in molecular calculations, where the integrals have to be evaluated about different nuclear centers. For atoms, however, integrals using STO and GTO basis sets are relatively easily computed. The two basis sets are quite different when it comes to how many basis elements are required. For example, Figure 2 shows the convergence in total energy for the case of $\mathrm{He} 1 s^{2}$, both at zero field and at $\beta_{Z}=0.05$ [where it has the quantum numbers $\left.\left(M_{S}, \pi_{z}, M\right)=(0,+1,+0)\right]$. The most salient feature in Figure 2 is the fact that the GTO computations require about five times as many basis elements as the STO for the same level of accuracy, for both zero and nonzero field. This poor convergence property for the GTO basis set can be understood in two ways: The first reason for the improved quality of the STO basis is the

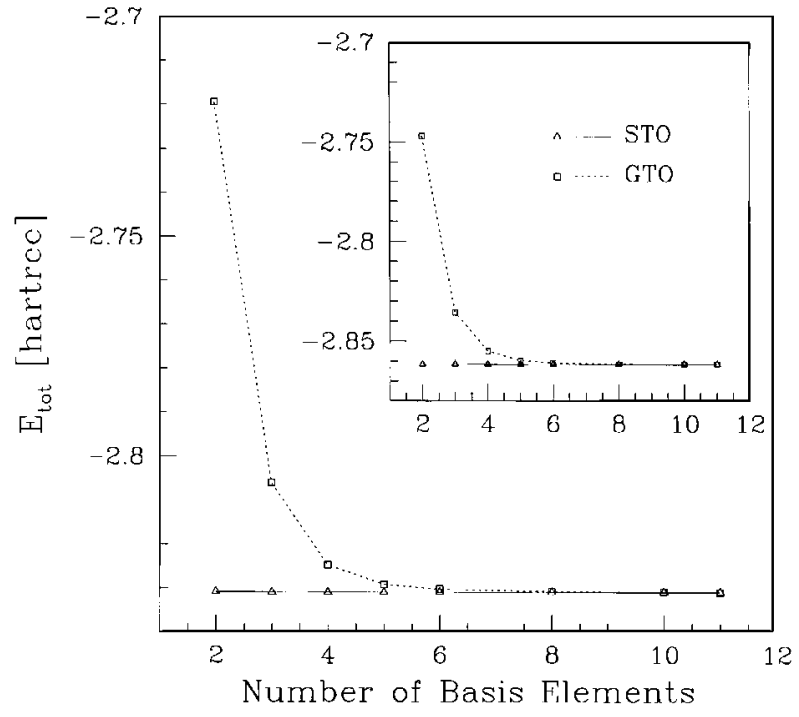

FIGURE 2. A comparison between the GTO and STO basis sets for the $\mathrm{He} 1 s^{2}$ state. The main figure is at $\beta_{Z}=.05$, while the inset is for zero field. All calculations were fully optimized over the orbital exponents.

fact that STOs are, by construction, solutions to the field-free hydrogen atom. It is therefore not particularly surprising that the STO basis elements outperform the GTO ones. The second consideration (albeit related to the first) is that the GTO basis elements do not have the correct behavior near the nucleus. If one factors out the leading contribution to $R(r)$,

$$
R(r)=r^{n-1} p(r) ;
$$

then the radial part of the hydrogenic Schrödinger equation leads to the condition

$$
\left.\frac{1}{p} \frac{d p(r)}{d r}\right|_{r=0}=-\frac{Z}{l+1} .
$$

This relation is known as the electron-nucleus cusp condition [31]. A similar result can be shown for the electron-electron cusp,

$$
\left.\frac{1}{p\left(r_{i j}\right)} \frac{d p}{d r_{i j}}\right|_{r_{i j}=0}=\frac{1}{2(l+1)} .
$$

Physically, the meaning of Eqs. (30) and (31) is that the singularities in the Coulomb potential are canceled by singularities in the kinetic energy. The GTO basis, unfortunately, cannot satisfy these cusp conditions, and one needs to add further basis elements to further improve the incorrect short- 
range behavior inherent in these basis elements. The STO basis set explicitly satisfies the electronnuclear cusp condition. While the GTO basis elements more closely resemble Landau orbitals, the failure to match the correct behavior at the nucleus becomes more acute as the field strength is increased, since atoms tend to shrink as they are subjected to stronger fields. In general, as Figure 2 suggests, we find that STOs outperform GTOs at all field strengths that we have studied. The electron-electron cusp condition requires that our wave function contain two-body terms, which our simple HF wave function cannot satisfy, no matter what basis set we choose.

\section{Basis-set Truncation Error}

One issue that arises in working with STO (or similar) basis-set representations for the atomic wave function is the uncertain nature of convergence to the HF variational energy. Ideally, one simply adds more basis elements or optimizes the exponents of the basis further until a limiting value is achieved. In practice, however, one encounters limitations in the amount of resources available, either in time or computer memory. The most time-consuming part of a basis-set HF calculation is the computation of the electron-electron matrix elements. For $N_{b}$ basis elements, approximately $\left(N_{b}\right)^{4}$ such matrix elements need to be evaluated. Figure 3 shows a typical set of calculations to get the lowest possible HF energy for the $1 s 2 p_{-1}$ state of $\mathrm{He}$ at $\beta_{Z}=1$. Note that convergence is achieved when the number of basis elements is greater than 35 (and the maximum $l$ used [in the spherical harmonics], $l_{\text {max }}$, is greater than 13). For more highly excited states (or outer-shell electrons in larger atoms), one must generally increase $l_{\max }$ further, which also increases $N_{b}$. We used $N_{b} \leq 70$ in all of our calculations presented here, which introduces a truncation error. This truncation error increases with both $Z$ and $\beta_{Z}$ and is quite difficult to estimate in any systematic fashion. As an example of the basis-set size, consider Figure 4, which shows the average number of basis elements $\left\langle N_{b}\right\rangle$ and $\left\langle l_{\max }\right\rangle$ for the first two excited states of helium having $M=0$ and $M=$ -1 . The more highly excited states feel a much larger effective magnetic field (since the Lorentz force increases relative to the Coulomb force with increasing radius of the atomic orbital), resulting in much larger (and more computationally intensive) basis-set sizes. Note that the overall size of

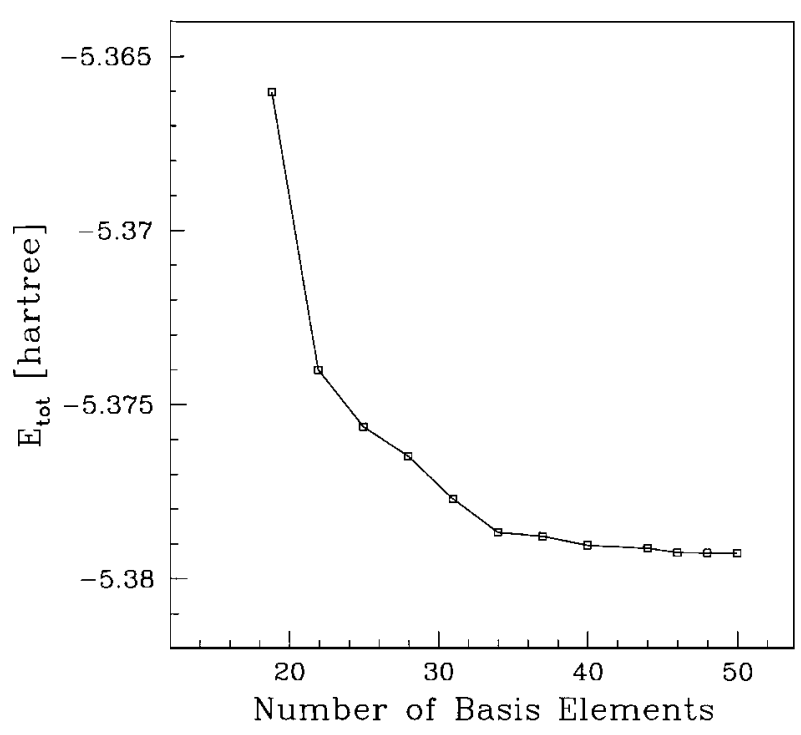

FIGURE 3. Saturation of basis elements for $\mathrm{He} 1 s 2 p_{-1}$ at $\beta_{Z}=1$.

the basis set does not necessarily increase uniformly with field strength; instead, we have to face a ceiling of the number of basis elements that can be computed in a reasonable length of time. Since a smaller number of basis elements are usually optimized, the final number of basis elements often depends on the quality of this primary optimization step.

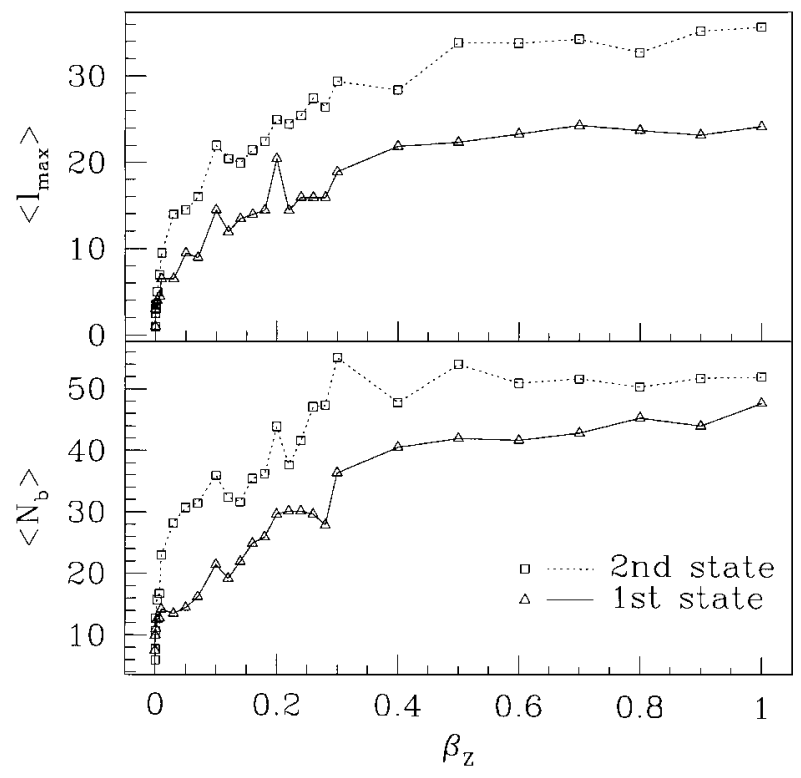

FIGURE 4. Average saturated basis-set size for the first two excited states of helium with $M=0$ and $M=-1$ as a function of field strength. 


\section{Hartree-Fock Results for Selected First-row Atoms}

Extensive tables of our HF total energies are included in [23]. In this section, we review the implications of these results and compare with previous works, where applicable.

\section{GROUND-STATE QUANTUM TRANSITIONS}

Tables I-VIII of [23] contain the total energy as a function of magnetic field strength for the low-lying electronic states of $\mathrm{H}^{-}, \mathrm{He}, \mathrm{Li}$, and $\mathrm{C}$ and many excited states of neutral He. We also list, for $\mathrm{H}^{-}$and $\mathrm{He}$, the results from other methods of calculation. For $\mathrm{Li}$ and $\mathrm{C}$, no comparisons are made, as we have found no computations performed in this range of magnetic fields for atoms with more than two electrons. As far as we know, these are the first calculations for these atoms for intermediate magnetic field strengths.

Ultimately, we wish to understand the spectral properties of light atoms in this range of magnetic fields, where many astrophysical applications can be found. Figure 5, therefore, shows the computed energy spectrum for the two electron atoms that we have considered thus far. The HF calculations of Thurner et al. [18], $E_{T h}$, are always slightly higher than the present results. This discrepancy is

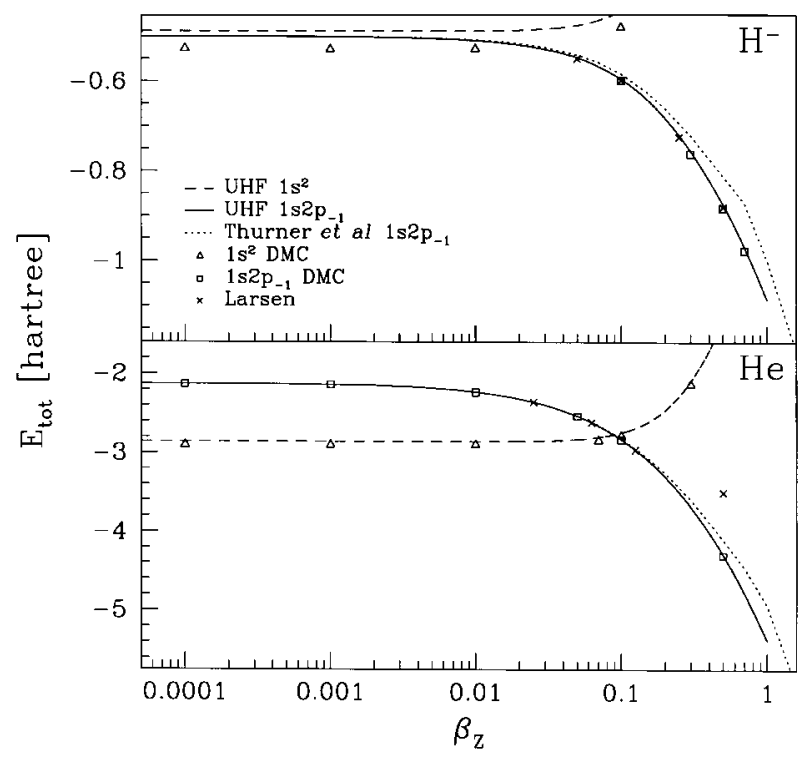

FIGURE 5. HF total energies for low-lying electronic states as a function of field strength for $\mathrm{H}^{-}$and $\mathrm{He}$. due to the ansatz used in the Thurner et al. method, where the wave function was expanded in spherical harmonics for small fields and Landau-like orbitals for large fields (see subsection Difficulties of the Magnetized Coulomb Problem). The two expansions meet in the intermediate range of field strength. Note that the $\mathrm{H}^{-}$comparison between the two HF methods, shown in Figure 1, demonstrates that the results of Thurner et al. are getting better as the field increases. The convergence of the two HF methods as we enter the superstrong regime is expected, since the assumption of cylindrical symmetry becomes more accurate as we approach the adiabatic regime. The variational energies of Larsen [21], $E_{L a r}$, which include electron correlation, are slightly lower than our HF numbers, as expected, and match very well with previous QMC calculations [22], which used GTO HF trial wave functions. Note that HF does not obtain the correct ground state for $\mathrm{H}^{-}$at small fields. The electron correlation (the difference between the HF and the exact energies) is sufficiently large to reorder the spin singlet and triplet states. At zero field, it has been shown that $\mathrm{H}^{-}$has only a single bound state [32], while in nonzero fields (even infinitesimally small), there are an infinite number of bound states [33]. This sudden plethora of bound states arises from the fact that the constant field pins the extra electron and thus enhances the attraction between the neutral atom and the additional electron. The QMC results [22] shown for the $1 s^{2}$ state are exact, due to the bosonic nature of the spatial component of the singlet wave function. We will discuss the QMC results and make further comparisons in later sections.

The case of He, a second bench mark of our calculations, is also shown in Figure 5, where we again compare our results with those of Thurner et al., Larsen, and the QMC calculations. Again, note that the numerical quadrature HF approach [18] is clearly inferior to our calculations and again improves as the field strength gets very large, near $\beta_{Z} \geq 1$. Also note that the correlation energy is much larger for the singlet state than for the triplet, since the singlet state is much more compact than the triplet. We will consider more highly excited states for neutral He in subsection The Spectrum of Neutral He.

As one would expect, as the field is increased, the ground state of the system becomes spinpolarized in order to minimize the Zeeman energy and reduce electron repulsion. For the two-electron systems considered thus far, there is only one such 
transition, from a spin singlet $(S=0)$ to a spin triplet $(S=1)$. This transition occurs near $\beta_{Z} \simeq$ 0.02 for $\mathrm{H}^{-}$and $\beta_{Z} \simeq 0.1$ for $\mathrm{He}$ and is also a transition in $M$, but not in $z$-parity. This type of transition is not difficult to understand. As the field gets larger, the system tends to shrink, and by raising the angular momentum of the most exterior electron, the atom is able to increase the electronic separation. Figure 6 shows the energy of the first four electronic states of neutral Li. In this case, there are two transitions: The first near $\beta_{z} \simeq$ 0.01 is a transition to a state of lower Zeeman energy ( $M=0$ to $M=-1, \pi_{z}$ unchanged, and $M_{S}$ unchanged), while the second, near $\beta_{Z} \simeq 0.16$, is the expected transition to a completely spin polarized state ( $M$ is unchanged, but $\pi_{z}=+1$ to $\pi_{z}=-1$, and $M_{S}=-1 / 2$ to $\left.M_{S}=-3 / 2\right)$. A similar plot can be drawn for $\mathrm{C}$, also shown in Figure 6 . In the case of $C$, however, there are two spin transitions. The first, near $\beta_{Z} \simeq 0.005$, from $M_{S}=-1$ to $M_{S}=-2$ (with $M=-1$ to $M=-2$, and $\pi_{z}$ from -1 to +1 ), and the second, at $\beta_{Z} \simeq$ 0.18 , to the completely spin-polarized state, $M_{S}=$ -3 (with both $M$ and $\pi_{z}$ unchanged). Figure 7 displays the ground state quantum numbers for the entire series of transitions, as determined by our HF calculations.

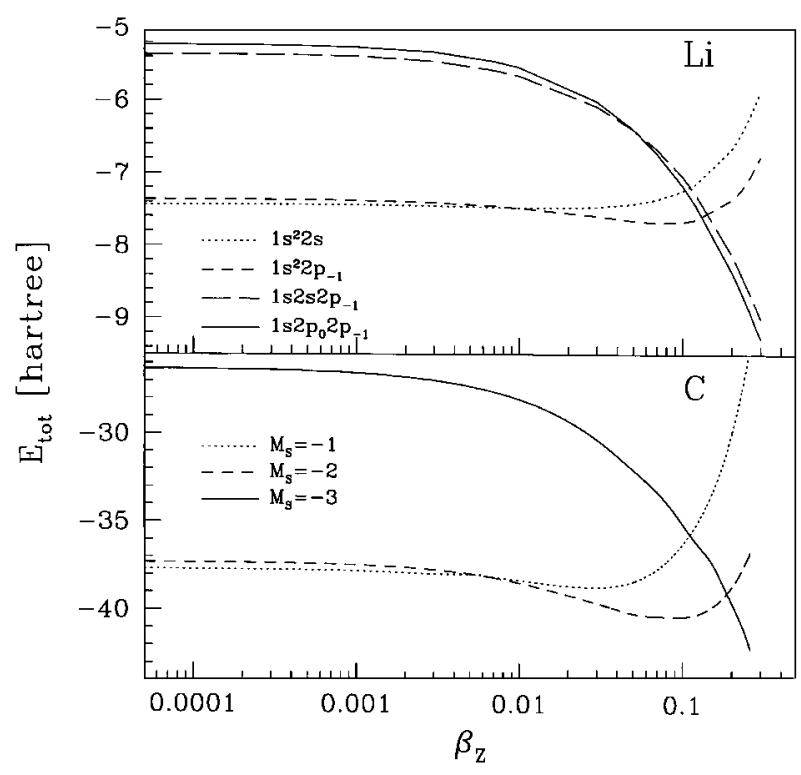

FIGURE 6. HF total energies for low-lying $\mathrm{Li}$ and $\mathrm{C}$ states as a function of magnetic field strength.

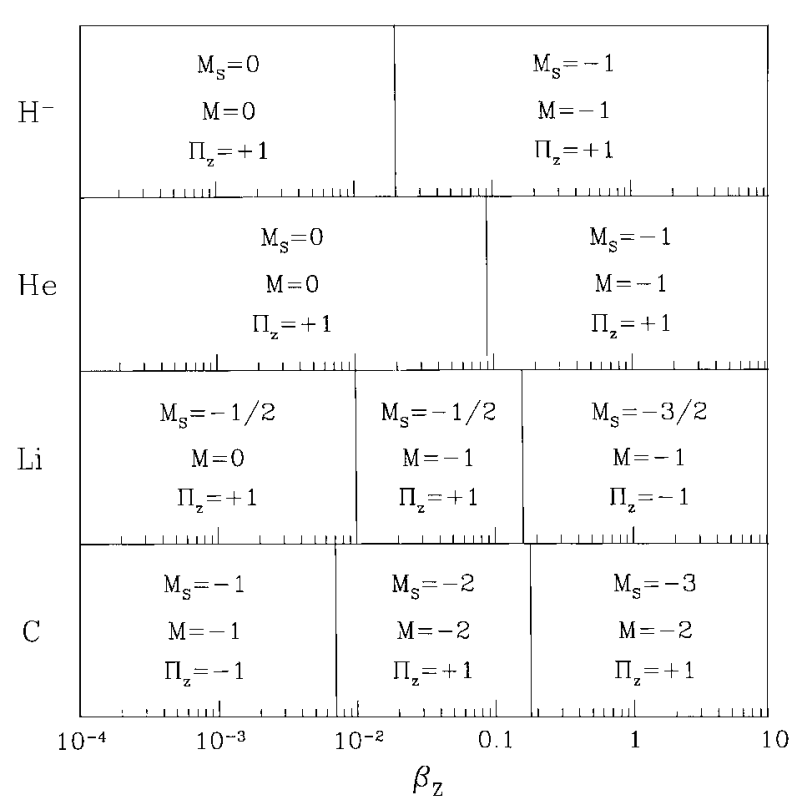

FIGURE 7. Ground-state quantum numbers for $\mathrm{H}^{-}$, $\mathrm{He}, \mathrm{Li}$, and $\mathrm{C}$ as a function of magnetic field strength.

\section{ELECTRON DENSITY}

Just as for the two-electron systems, the larger atoms increase the rotational energy of the electrons in order to decrease the electron repulsion. We can visualize these changes by examining the electronic density for various atomic states as a function of field strength. The electron density, $\rho(\mathbf{r})$, is easily computed from the individual orbitals:

$$
\rho(\mathbf{r})=\sum_{a=1}^{N}\left|\phi_{a}(\mathbf{r})\right|^{2} .
$$

Figure 8 shows the electron density profiles for the ground state of each atom at several different values of the field strength. Atoms that in small fields are almost spherically symmetric acquire very compact butterfly or needlelike shapes as the field strength increases. Also note the needlelike structure of the final completely spin-polarized ground state at the largest magnetic field value for each atom. The excited states show much the same behavior as a function of field strength. Figure 9 shows a comparison between selected excited states of neutral helium, at zero field (top) and $\beta_{Z}=0.03$ (bottom). The strong field plot is on a smaller scale (factor of two), and the contours are drawn at the same values of the electron density. 


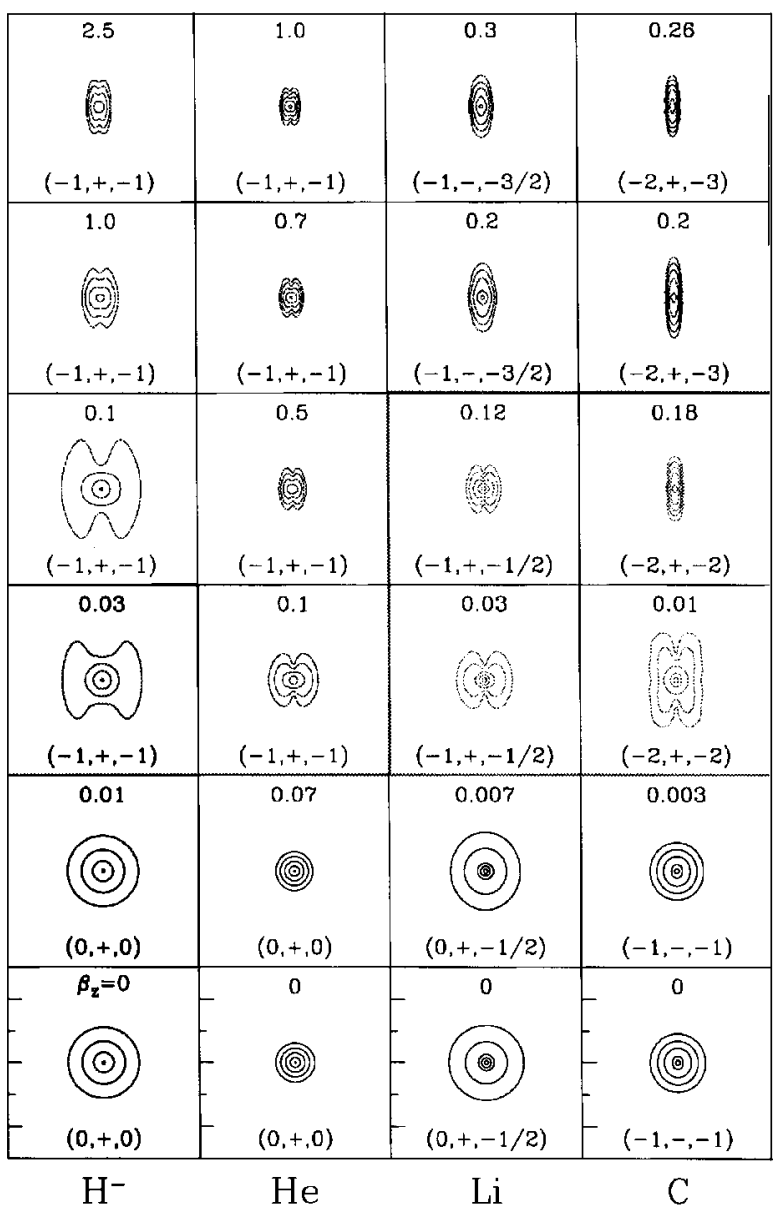

FIGURE 8. The electron density of the ground state of $\mathrm{H}^{-}, \mathrm{He}, \mathrm{Li}$, and $\mathrm{C}$ as a function of magnetic field strength. Numbers in parentheses indicate the values of the quantum numbers $\left(M, \pi_{z}, M_{S}\right)$.

Note the dramatic effect of the field on the shape of the atom, similar to the ground-state shapes shown in Figure 8, and the change in order of the excited states according to energy. Based upon this radical change in the physical structure of the atomic states, one would also expect strong changes in the spectral properties, which we now discuss for helium.

\section{THE SPECTRUM OF NEUTRAL He}

In an effort to compute the spectral features of helium in a magnetic field, we carried out extensive HF calculations of several excited states. Figure 10 shows the results for the first two states of $\left.\left.M_{S}=0, \quad M \in[0,-1,-2,-3], \pi_{z} \in\right]+1,-1\right]$ symmetry. The more familiar zero-field quantum

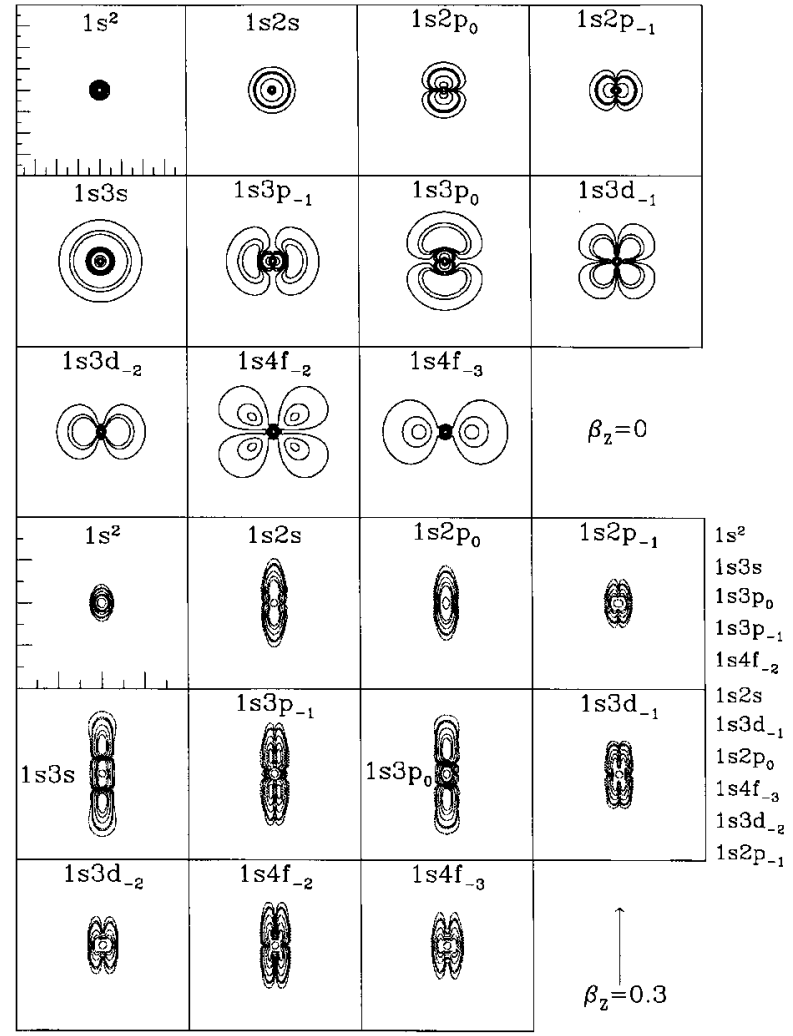

FIGURE 9. A comparison of the HF electron density for some excited states of helium, at zero field (top) and $\beta_{Z}=.3$ (bottom). Note that the scale has been reduced by two for the strong field plot. Each large tick mark is 10 bohr radii. The states at zero field are ordered energetically from left to right, top to bottom. The correct ordering at $\beta_{Z}=.3$ is given by the key on the right side of the lower plot.

numbers are also included. The points represent the calculations of Thurner et al. [18]. Without exception, we achieve significantly lower variational energies, especially in the range $\beta_{Z}>0.1$. Unfortunately, although the present results are much better upper-bound estimates of the atomic energies, we also have significant basis-set truncation errors, as we discussed in subsection Basis-set Truncation Error. These nonsystematic errors result in a very poor estimate of the helium spectrum for $\beta_{Z}>0.1$, precisely in the region of most interest for magnetized white dwarf spectra. Thus, although basis sets allow for an efficient and reasonably accurate determination of the variational energy, the uncertainty arising from truncation prevents an accurate determination of the spectral features. It is primarily for this reason that we consider, in subsequent sections, the application of 


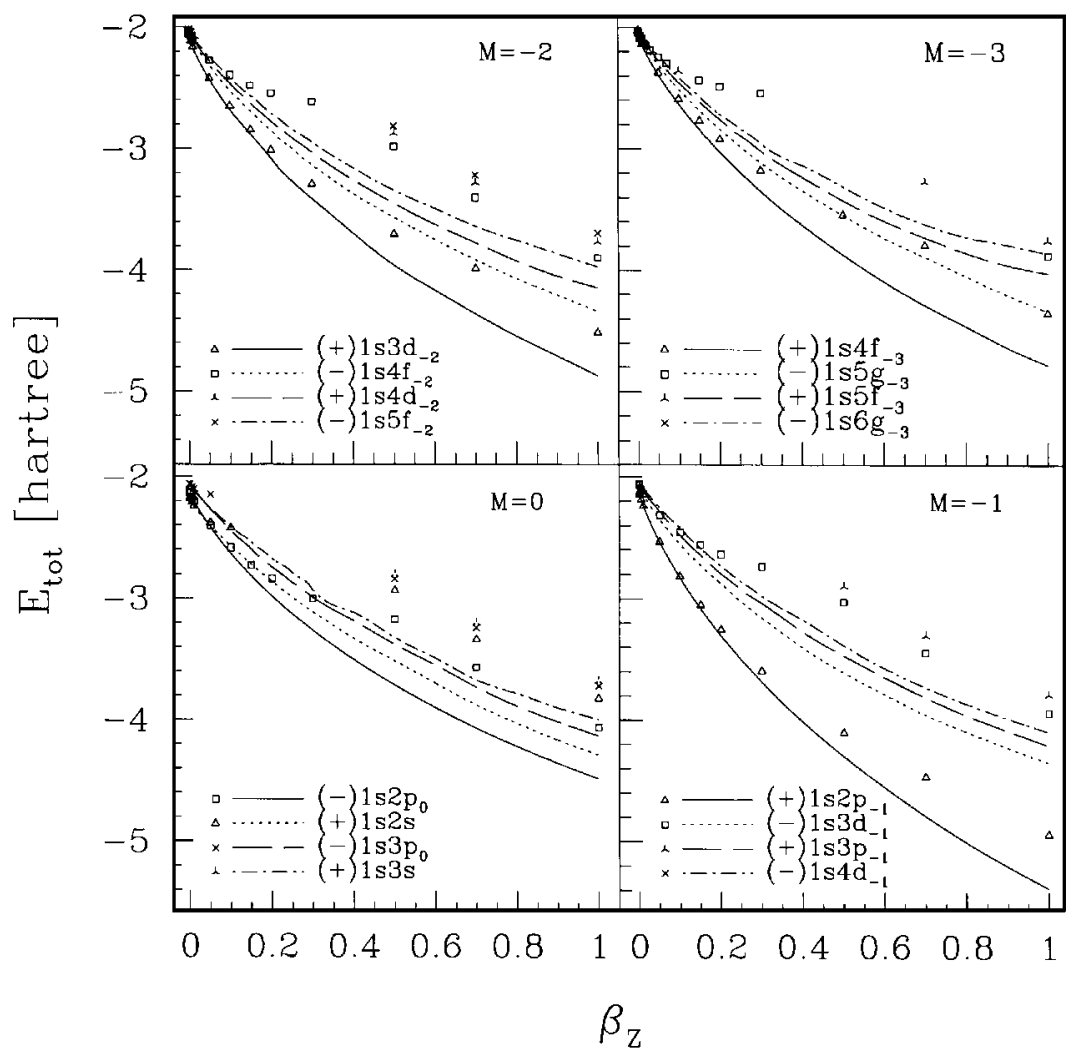

FIGURE 10. Excited-state energies of spin-polarized helium. Lines are the present HF results, while points are those of Thurner et al. [18]. The sign in parentheses that precedes the zero field quantum numbers is the $z$-parity, $\pi_{z}$.

QMC methods for magnetized helium. With QMC, we shall be able to simultaneously deal with the basis-set truncation error and the correlation effects.

\section{CONCLUSIONS}

Although we have presented results of unprecedented accuracy for magnetized multielectron atoms in strong magnetic fields, the preceding section makes clear one of the leading failings of the basis-set HF approach. It is quite extraordinarily difficult to achieve a saturated basis set when the applied field requires many high-order spherical harmonics. Even at zero field, the generation of highly optimized basis sets for atomic and molecular calculations has been, and continues to be, a field of its own within computational chemistry. ${ }^{\dagger}$ The basis-set truncation error can be reduced with improvements in both algorithm and computational resources that allow for the calculation and optimization of larger basis sets.

\footnotetext{
${ }^{\dagger}$ See [34] for a review of a variety of Gaussian basis sets in common use.
}

Another problem with the current HF treatment is the lack of many-body effects within this meanfield approach. No amount of improvement in the basis set will make up for the many-body correlation effects, which cannot be taken into account by a single Slater determinant consisting of singleparticle orbitals. In the following sections, we will concentrate on highly accurate QMC solutions to two-electron magnetized systems. The QMC techniques will enable us to simultaneously resolve both of these difficulties and accurately determine the spectrum of magnetized helium.

\section{Stochastic Methods in Strong Magnetic Fields}

\section{INTRODUCTION}

We have already noted that the spectrum of magnetized helium, as determined by a mean-field approach, is unsatisfactory. The importance of many-body effects remains unknown. One might think that the many-body effects absent in HF are 
relatively unimportant for a two-electron system, but this assumption may be misleading as an applied field causes an atom to shrink, further localizing the electrons. We do know, however, that the error from inadequate basis sets is quite severe. In this section, we introduce a method that simultaneously remedies the basis-set problem and includes many-body effects, using a collection of stochastic random walks to solve the Schrödinger equation in imaginary time $t$ (i.e., after performing a Wick rotation). After presenting the method, we use this quantum Monte Carlo (QMC) technique to evaluate the HF calculations presented in the previous section and compute an updated spectrum of magnetized helium.

Since the system Hamiltonian [Eq. (1)] can be written as $\hat{\mathbb{T}}=\hat{\mathbb{H}}_{\mathscr{R}}(\mathscr{R})+\hat{\mathbb{H}}_{S}(\Sigma)$ (where $\mathscr{R}=\left(\mathbf{r}_{1}\right.$, $\left.\ldots, \mathbf{r}_{i}, \ldots, \mathbf{r}_{N}\right)$ denotes a point in configuration space (a Cartesian manifold of dimension $3 N$ ), and $\left.\Sigma=\left(s_{1}, \ldots, s_{i}, \ldots s_{N}\right)\right)$, the many-body wavefunction $\Psi(\mathscr{R}, \Sigma)$ can be written as a product of a coordinate and a spin function (or a linear combination of such products):

$$
\Psi(\mathscr{R}, \Sigma)=\Phi(\mathscr{R}) \otimes \chi(\Sigma) .
$$

We want to construct $N$-fermion eigenstates of $\hat{H}$ that are also eigenfunctions of the total spin $\hat{S}^{2}$,

$$
\hat{S}^{2} \Psi(\mathscr{R}, \Sigma)=\hbar^{2} S(S+1) \Psi(\mathscr{R}, \Sigma),
$$

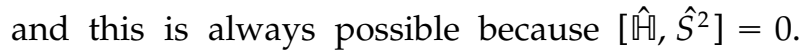
Thus, the configuration part $\Phi(\mathscr{R})$ must have the right symmetry in order to account for the Pauli principle. Moreover, $\Phi$ possesses the property of Fock's cyclic symmetry,

$$
\left(\mathbb{1}-\sum_{j=k+1}^{N} \hat{P}_{k j}\right) \Phi=0,
$$

where, in this case, $\hat{P}_{k j}$ refers to the transposition of particle coordinates $\mathbf{r}_{k}$ and $\mathbf{r}_{j}$. This last condition is a very useful one for testing the symmetry of a given coordinate function.

For a given total spin $S$, we are thus left with the task of solving a stationary many-body Schrödinger equation $\hat{\mathbb{H}} \mathscr{R} \Phi(\mathscr{R})=E \Phi(\mathscr{R})$, where $\Phi(\mathscr{R})$ satisfies the symmetry constraint discussed above. In the following discussions, therefore, we shall drop the subscript on $\hat{\mathbb{H}}_{\mathscr{R}}$.

\section{FIXED-NODE OMC}

The most common approach in ground-state QMC calculations considers the ground-state wave function to be real. In the absence of external fields (the subject of this section), the wave function can always be taken to be a real function. For electronic systems, the total wave function must be antisymmetric under the simultaneous interchange of spatial and spin coordinates. We will be concerned with both spin symmetric and spin antisymmetric states-hence, the spatial part of the wave function may be either antisymmetric or symmetric, respectively. The ground state of an antisymmetric spatial wave function will be divided into equal regions of positive and negative sign. The variance of a Monte Carlo average determined for such a system can be very large. To avoid this situation, we turn to the idea of importance sampling and multiply our desired groundstate wave function, $\Phi(\mathscr{R}, t)$, by a guiding ${ }^{\ddagger}$ wave function $\psi_{T}$,

$$
f(\mathscr{R}, t)=\Phi(\mathscr{R}, t) \psi_{T}(\mathscr{R}),
$$

where $t$ denotes imaginary time. Our sampled distribution will then be $f$, and we impose the constraint that, within each nodal region, $f$ is positive definite, thus forcing $\Phi$ to have the same nodal surface as $\psi_{T}$. This fixed-node approximation removes the "sign problem" and is then the starting point for most applications of QMC. The emphasis is then placed on obtaining the best possible trial wave functions, with the optimal representation of the true nodal structure. For an applied magnetic field, however, we have to confront the possibility that our wave function may not be real-valued.

\section{FIXED-PHASE OMC}

When a magnetic field is applied, eigenfunctions of $\hat{\mathbb{H}}$ will, in general, be complex-valued because of the explicitly broken time-reversal symmetry. This fact, upon first viewing, seems unremarkable, but does pose a problem, as the simple square of the wave function can no longer be interpreted as a probability. The next step, in the fixed-phase approach [35], is to break the wave function apart,

$$
\Phi(\mathscr{R})=|\Phi(\mathscr{R})| e^{i \varphi(\mathscr{R})},
$$

\footnotetext{
\# There is a distinction often made between the wave functions used to determine the local energy (the trial wave function) and that used for importance sampling (the guiding function). For the current section, the same function $\psi_{T}$ is used for both purposes.
} 
where we explicitly separate out the probability density in the form of the modulus of the wave function and the phase $\varphi(\mathscr{R})=-i \ln [\Phi /|\Phi|]$. Recall that the atomic Hamiltonian, in atomic units, is given by (the generalization to the molecular case is straightforward)

$$
\hat{\mathscr{H}} \Phi=\sum_{j=1}^{N}\left[\frac{1}{2} \Pi_{j}^{2}-\frac{Z}{r_{j}}+\sum_{k<j} \frac{1}{r_{j k}}\right] \Phi .
$$

We take the energy as a functional of the modulus and the phase,

$$
E[|\Phi|, \varphi]=\frac{\langle\Phi|\hat{\mathbb{H}}| \Phi\rangle}{\langle\Phi \mid \Phi\rangle} .
$$

We then consider independent variations in $|\Phi|$ and $\varphi$, which leads us to two independent equations:

$$
\begin{aligned}
& \operatorname{Re}\left\{e^{-i \varphi}(\hat{\mathscr{H}}-E) \Phi\right\}=0, \\
& \operatorname{Im}\left\{e^{-i \varphi}(\hat{\mathfrak{H}}-E) \Phi\right\}=0 .
\end{aligned}
$$

These equations are readily evaluated,

$$
\begin{gathered}
{\left[-\sum_{j=1}^{N} \frac{1}{2} \nabla_{j}^{2}+V_{\text {eff }}(\mathscr{R})\right]|\Phi|=E|\Phi|,} \\
\sum_{j=1}^{N} \nabla_{j} \cdot\left[|\Phi|^{2}\left(\mathscr{A}_{j}(\mathscr{R})+\mathbf{A}\left(\mathbf{r}_{j}\right)\right)\right]=0,
\end{gathered}
$$

where $\mathscr{A}_{j}(\mathscr{R})=\nabla_{j} \varphi$ and the effective potential is given by

$$
\begin{aligned}
V_{\text {eff }}(\mathscr{R})=\sum_{j=1}^{N}\left(\frac { 1 } { 2 } \left[\mathscr{A}_{j}(\mathscr{R})\right.\right. & \left.+\mathbf{A}\left(\mathbf{r}_{j}\right)\right]^{2} \\
& \left.-\frac{Z}{r_{j}}+\sum_{k<j} \frac{1}{r_{j k}}\right) .
\end{aligned}
$$

In Eq. (42), we have a Schrödinger equation for the modulus of the wave function (albeit with an effective potential), coupled to another equation [Eq. (43)] which resembles the continuity expression for the probability density. Ideally, one would solve both equations simultaneously; instead, we assume the phase, $\varphi$, to be a fixed quantity, $\varphi_{T}$ (hereafter referred to as the trial phase), and then solve the resulting Schrödinger equation for the modulus. This assumption is the basis of the fixed-phase method developed by Ortiz et al. [35]. The trial phases must satisfy some mathematical constraints. The phases should, e.g., conserve the symmetries of the Hamiltonian (unless some are spontaneously broken) and particle statistics. We now take the time to explore this method in detail, since much of the formalism and discussion will be relevant for our later work.

Once a trial phase has been obtained, the equation for the modulus remains to be solved. Our method will rely on the use of Monte Carlo techniques (identical to the fixed-node methodology) to evaluate the multidimensional integral equation equivalent of the Schrödinger equation. We first write the Schrödinger equation in imaginary time,

$$
\begin{aligned}
-\partial_{t}|\Phi(\mathscr{R}, t)|=\left(-\frac{1}{2} \sum_{j=1}^{N} \nabla_{j}^{2}+\right. & \left.V_{\mathrm{eff}}(\mathscr{R})-E_{T}\right) \\
& \times|\Phi(\mathscr{R}, t)|,
\end{aligned}
$$

where we have introduced $E_{T}$ to shift the energy spectrum (for reasons which we will return to below). For the remainder of this section, we will take the quantity in parentheses in Eq. (45) as our new effective Hamiltonian, $\hat{H}$; this can be considered as the fixed-phase Hamiltonian. Note that Eq. (45) can be interpreted as a diffusion equation with a branching term, given by $V_{\text {eff }}(\mathscr{R})-E_{T}$. An expansion in exact eigenstates of the Hamiltonian, $\left\{\phi_{i}\right\}$,

$$
\Phi(\mathscr{R}, t)=\sum_{i} b_{i} e^{-\left(E_{i}-E_{T}\right) t} \phi_{i}(\mathscr{R}),
$$

reveals that, if $b_{0} \neq 0$, and $E_{T}$ is adjusted to equal the ground-state energy, then the asymptotic steady-state solution is the ground state,

$$
\Phi(\mathscr{R}, t \rightarrow \infty)=b_{0} \phi_{0}(\mathscr{R}) .
$$

This property is extremely useful and is the key feature of the projection in imaginary time that we will use in solving Eq. (45). It is difficult to know, before solving the problem, how to adjust the trial energy, $E_{T}$, such that $E_{T}=E_{0}$. Another difficulty is that the potential is singular at coincident points $\left(\mathbf{r}_{i}=\mathbf{r}_{j}\right)$, which causes large fluctuations in the value of the branching term in Eq. (45). A way around this difficulty can be found by multiplying the Schrödinger equation for $\Phi$ by a real-valued positive guiding function $\psi_{T}$ and rearranging the terms such that

$$
\begin{aligned}
-\partial_{t} F(\mathscr{R}, t)=-\frac{1}{2} \sum_{j=1}^{N} \nabla_{j}^{2} f & +\sum_{j=1}^{N} \nabla_{j} \cdot\left(f \nabla_{j} \ln \psi_{T}\right) \\
& +\left(E_{L}(\mathscr{R})-E_{T}\right) f, \quad
\end{aligned}
$$


where $f=|\Phi(\mathscr{R}, t)| \psi_{T}(\mathscr{R})$, and $E_{L}=\psi_{T}^{-1} \hat{H} \psi_{T}$ is the local energy of the trial wave function Equation (48) is a diffusion equation with both drift and branching terms. The drift is governed by the "quantum force," $\nabla \ln \psi_{T}$, while the branching is controlled by the difference between the local and trial energies. The advantage of using the trial wave function is that the fluctuations in the branching term can be greatly reduced. The longtime distribution for $f$ is then given by

$$
f(\mathscr{R}, t \rightarrow \infty)=\left|b_{0} \phi_{0}(\mathscr{R})\right| \psi_{T}(\mathscr{R}) .
$$

Let us now consider the best way of solving the so-called master equation, Eq. (48).

The Green's function,

$$
G\left(\mathscr{R}, \mathscr{R}^{\prime} ; \tau\right)=\psi_{T}(\mathscr{R})\left\langle\mathscr{R}\left|e^{-\pi\left(\hat{H}-E_{T}\right)}\right| \mathscr{R}^{\prime}\right\rangle \psi_{T}^{-1}\left(\mathscr{R}^{\prime}\right),
$$

for the master equation also satisfies Eq. (48). If we could analytically solve Eq. (48) for the Green's function, than our problem would be solved. The physical interpretation of $G$ is that it represents the probability of a particle moving from $\mathscr{R}^{\prime}$ to $\mathscr{R}$ in time $\tau$,

$$
f(\mathscr{R}, t+\tau)+\int d \mathscr{R}^{\prime} G\left(\mathscr{R}, \mathscr{R}^{\prime} ; \tau\right) f\left(\mathscr{R}^{\prime}, t\right) .
$$

We can obtain an approximate form for the Green's function by assuming that the quantum force and the local energy change very little in the move from $\mathscr{R}^{\prime}$ to $\mathscr{R}$. In this case, the approximate Green's function for Eq. (48) is given by

$$
\begin{aligned}
G\left(\mathscr{R}, \mathscr{R}^{\prime} ; \tau\right) \simeq & G_{d}\left(\mathscr{R}, \mathscr{R}^{\prime} ; \tau\right) G_{b}\left(\mathscr{R}, \mathscr{R}^{\prime} ; \tau\right), \\
= & (2 \pi \tau)^{-3 N / 2} e^{-\left(\mathscr{R}-\mathscr{R}^{\prime}-\tau \mathrm{F}_{Q}(\mathscr{R})\right)^{2} / 2 \tau} \\
& \times e^{-\tau\left[\left(E_{L}(\mathscr{R})+E_{L}\left(\mathscr{R}^{\prime}\right)\right) / 2-E_{T}\right]}
\end{aligned}
$$

where $\mathbf{F}_{Q}=\nabla \ln \psi_{T}$ is the quantum force. Corrections to this short-time approximation are of order $\tau^{2}$. Note that the first term describes a Gaussian whose mean is drifting according to an effective velocity determined by the quantum force, pushing the particle toward regions of increasing $\psi_{T}$. The second term is simply a rate (or branching) term, which controls how fast the configuration (or "walker") reproduces. Equation (52) governs the dynamics of our random walks. $E_{T}$ is constantly adjusted to be equal to the estimated ground-state energy:

$$
E_{0}=\lim _{t \rightarrow \infty}\left\langle E_{L}(\mathscr{R})\right\rangle_{f}=\frac{\int d \mathscr{R} f(\mathscr{R}, t \rightarrow \infty) E_{L}(\mathscr{R})}{\int d \mathscr{R} f(\mathscr{R}, t \rightarrow \infty)} .
$$

One obtains the asymptotic distribution $f(\mathscr{R}, t \rightarrow$ $\infty)$ by repeated iterations of Eq. (51). An appropriate choice of the trial function will not only reduce the fluctuations in the branching term, but will also serve a crucial role in directing the walkers toward the important regions of phase space. By rewriting the Schrödinger equation in this fashion, we succeeded in mapping our quantum mechanical problem into a classical random walk.

There are several useful properties associated with the fixed-phase method. First, the method is variational, i.e., the resulting energy, $E_{0}$, obtained by solving the Schrödinger equation for the modulus is an upper bound to the exact energy and, for a prescribed trial phase $\varphi_{T}$, is the lowest energy consistent with this phase. A phase that satisfies Eq. (43), (continuity equation) for the exact modulus will lead to the exact solution of this many-fermion problem. If the wave function is real-valued, the fixed-phase method reduces to the fixed-node method, provided that the phase is taken to be a simple step function:

$$
\varphi(\mathscr{R})= \begin{cases}0 & \Phi(\mathscr{R}) \geq 0 \\ \pi & \Phi(\mathscr{R})<0 .\end{cases}
$$

There are several features of the method that are not, however, very desirable. First, one does not know, a priori, the quality of the trial phase. One can, however, systematically improve a given trial phase using projection techniques [36].

Second, this method, like the fixed-node approximation only provides a variational bound for the ground state of a particular symmetry. One could imagine, based upon Eq. (49), constructing a trial wave function that does not overlap the ground state and thus obtain convergence to a higher excited state. In practice, this trick is quite difficult. In the following section, we discuss a method for relaxing both of these constraints, which will allow us to determine the "exact" solution for both ground- and excited-state properties.

\section{RELEASED-PHASE QMC}

Now we discuss the possibility of calculating exact results in QMC, unencumbered by the fixed-node or fixed-phase restrictions. First, we 
note that time-step error can be eliminated by sampling the exact Green's function instead of the approximate form that we have already introduced, through writing the Green's function as an additional integral equation (Laplace transform). This method is known as the Green's function QMC (GFQMC) [37] or domain GFQMC. The time-step error, however, is an easily controllable approximation. We can simply reduce the time-step error below that of the statistical errors for the quantity of interest. Here, we are concerned with the uncontrolled approximations, namely, the unknown quality of the phase of the total wave function.

Zero-temperature quantum Monte Carlo (MC) methods typically begin with the choice of the trial wave function. Here, we are interested in ground and excited states, so we begin by choosing a basis of trial wave functions that represent our best (analytic) approximation to the spectrum of states that we wish to examine. In the correlation function MC method, one projects this basis with the exponential of the Hamiltonian operator using random walks. Matrices formed by the resulting autocorrelation functions evaluated during the random walk are then solved for the energy spectrum and other properties. The eigenvalues converge to the exact energies of the system in the limit of infinite imaginary time, but the variance of the energy grows both exponentially in time and excitation energy. Thus, in practice, this method is limited by the size of the system and the number of excitations of a given symmetry. Our presentation of the correlation function method is necessarily brief; see [38] for further details of the method. Here, we review the formalism required for complex Hamiltonians. A more complete discussion of this released-phase approach can be found in [24].

Given a basis set $\left\{f_{j}\right\}$ of $N_{m}$ linearly independent states which approximate the lowest-energy states of our system, the exact eigenfunctions can be approximated in terms of this basis:

$$
\Phi_{i}(\mathscr{R}, 0)=\sum_{j=1}^{N_{m}} d_{i j} f_{j}(\mathscr{R}) .
$$

The variational theorem asserts that upper bounds [39] to the exact energies, $E_{i}^{e x}$, can be determined by finding the stationary points of the Rayleigh quotient,

$$
\Lambda_{i}(0)=\frac{\int d \mathscr{R} \Phi_{i}^{*}(\mathscr{R}, 0) \hat{\mathscr{H}} \Phi_{i}(\mathscr{R}, 0)}{\int d \mathscr{R} \Phi_{i}^{*}(\mathscr{R}, 0) \Phi_{i}(\mathscr{R}, 0)},
$$

with respect to the coefficients $d_{i j}$ ( $\hat{\mathbb{U}}$ is the complex Hamiltonian of the system under consideration). The size of the basis set $N_{m}$ determines the maximum number of excited-state energies that can be bounded.

Let us use the imaginary time-density matrix to find a new basis,

$$
\left\{\tilde{f_{i}}(\mathscr{R}, t)\right\}=\left\{e^{-t \hat{\mathbb{H}} / 2} f_{i}(\mathscr{R}, 0)\right\},
$$

a basis in which, as $t$ increases, the higher-energy components have been reduced relative to the lowest-energy states. If we make $\Lambda_{i}(t)$ stationary with respect to the expansion coefficients $\left\{d_{i j}(t)\right\}$, we arrive at a matrix equation for each eigenvector $\mathbf{d}_{i}^{\mathrm{T}}=\left(d_{i 1}, d_{i 2}, \ldots d_{i N_{m}}\right)$,

$$
\mathbf{H d}_{i}=\Lambda_{i}(t) \mathbf{S d}_{i},
$$

where the matrices $\mathbf{H}$ and $\mathbf{S}$ are given by

$$
\begin{aligned}
\mathbf{H}_{j k}(t) & =\int d \mathscr{R} d \mathscr{R}^{\prime} f_{j}^{*}(\mathscr{R})\left\langle\mathscr{R}\left|\hat{\mathbb{H}} e^{-t \hat{\mathbb{W}}}\right| \mathscr{R}^{\prime}\right\rangle f_{k}\left(\mathscr{R}^{\prime}\right), \\
\mathbf{S}_{j k}(t) & =\int d \mathscr{R} d \mathscr{R}^{\prime} f_{j}^{*}(\mathscr{R})\left\langle\mathscr{R}\left|e^{-t \hat{\mathbb{H}}}\right| \mathscr{R}^{\prime}\right\rangle f_{k}\left(\mathscr{R}^{\prime}\right) .
\end{aligned}
$$

Solving Eq. (58) allows us to project out the lowest $N_{m}$ energy states while simultaneously enforcing orthogonality through the generalized eigenproblem process.

To reduce the statistical variance in the Monte Carlo evaluation of $\mathbf{H}$ and $\mathbf{S}$, one again introduces importance sampling by multiplying and dividing by a guiding function $\psi_{G}$. We will assume that $\psi_{G}$ is normalized. The importance sampled Green's function is given by

$$
G\left(\mathscr{R}, \mathscr{R}^{\prime} ; t\right)=\psi_{G}(\mathscr{R})\left\langle\mathscr{R}\left|e^{-t \hat{\uplus}}\right| R^{\prime}\right\rangle \psi_{G}^{-1}\left(\mathscr{R}^{\prime}\right),
$$

where $\psi_{G}$ is a real-valued, nonnegative function. The matrices in terms of $G$ then become

$$
\begin{aligned}
\mathbf{H}_{j k}(t)= & \int d \mathscr{R} d \mathscr{R}^{\prime} F_{j}^{*}(\mathscr{R}) G\left(\mathscr{R}, \mathscr{R}^{\prime} ; t\right) \\
& \times E_{k}\left(\mathscr{R}^{\prime}\right) F_{k}\left(\mathscr{R}^{\prime}\right) \psi_{G}^{2}\left(\mathscr{R}^{\prime}\right), \\
\mathbf{S}_{j k}(t)= & \int d \mathscr{R} d \mathscr{R}^{\prime} F_{j}^{*}(\mathscr{R}) G\left(\mathscr{R}, \mathscr{R}^{\prime} ; t\right) \\
& \times F_{k}\left(\mathscr{R}^{\prime}\right) \psi_{G}^{2}\left(\mathscr{R}^{\prime}\right),
\end{aligned}
$$


where $F_{i}=f_{i} / \psi_{G}$ and $E_{i}=f_{i}^{-1} \hat{\mathfrak{H}} f_{i}$ is the local energy of the individual basis states. Note that $E_{i}$ is, in general, a complex-valued function of $\mathscr{R}$.

Because the Green's function is an exponential operator, we can expand it in terms of an Euclidean path integral:

$$
\begin{aligned}
G\left(\mathscr{R}, \mathscr{R}^{\prime} ; t\right)=\int d \mathscr{R}_{1} & \ldots d \mathscr{R}_{n-1} \\
& \times \prod_{j=1}^{n} G\left(\mathscr{R}_{j}, \mathscr{R}_{j-1} ; \tau\right),
\end{aligned}
$$

where $\mathscr{R}_{0}=\mathscr{R}^{\prime}, \mathscr{R}_{n}=\mathscr{R}$ (the complete path consists of $n$ individual steps), and $t=n \tau$. At sufficiently large $n$ (small $\tau$ ), we can write down accurate approximations to the Green's function and sample it with diffusion Monte Carlo.

During the random walk, we sample from a diffusion Green's function $G_{d}\left(R_{j}, R_{j-1} ; \tau\right)$ with a time interval $\tau$, iteratively constructing a tragectory of configurations $\left\{\mathscr{R}_{1}, \ldots, \mathscr{R}_{p}\right\}$ ( $p$ is the total length of the random walk), distributed according to the distribution

$$
\mathscr{P}\left(\mathscr{R}_{l}\right)=\psi_{G}^{2}\left(\mathscr{R}_{0}\right) \prod_{j=1}^{l} G_{d}\left(\mathscr{R}_{j}, \mathscr{R}_{j-1} ; \tau\right),
$$

where $l \leq n$. The matrices are evaluated over the course of each trajectory as

$$
\begin{aligned}
\mathbf{H}_{j k}(l \tau) & =\int d \mathscr{R}_{0} \ldots d \mathscr{R}_{l} \mathbf{h}_{j k}(l \tau) \mathscr{P}\left(\mathscr{R}_{l}\right), \\
\mathbf{S}_{j k}(l \tau) & =\int d \mathscr{R}_{0} \ldots d \mathscr{R}_{l} \mathbf{s}_{j k}(l \tau) \mathscr{P}\left(\mathscr{R}_{l}\right) .
\end{aligned}
$$

A single random walk can compute all of the matrix elements simultaneously; the correlation between the fluctuations in $\mathbf{H}$ and $\mathbf{S}$ will reduce the statistical error of the estimated eigenvalue. The estimates of the matrices are then given by comparing Eqs. (66) and (67) with Eqs. (62) and (63),

$$
\begin{aligned}
\mathbf{h}_{j k}(l \tau)= & \frac{1}{2(p-l)} \sum_{n=1}^{p-l} W_{n, n+l} F_{j}^{*}\left(\mathscr{R}_{n}\right) \\
& \times F_{k}\left(\mathscr{R}_{n+l}\right)\left[E_{k}\left(\mathscr{R}_{n+l}\right)+E_{j}^{*}\left(\mathscr{R}_{n}\right)\right],
\end{aligned}
$$

$\mathbf{s}_{j k}(l \tau)=\frac{1}{p-l} \sum_{n=1}^{p-l} W_{n, n+l} F_{j}^{*}\left(\mathscr{R}_{n}\right) F_{k}\left(\mathscr{R}_{n+l}\right)$, where

$$
\begin{aligned}
W_{n, n+l}=\prod_{i=1}^{l} G\left(\mathscr{R}_{n+i},\right. & \left.\mathscr{R}_{n+i-1} ; \tau\right) / \\
& G_{d}\left(\mathscr{R}_{n+i}, \mathscr{R}_{n+i-1} ; \tau\right) .
\end{aligned}
$$

Note that the weights $W_{n, n+l}$ will, in general, be complex-valued.

The eigenvalues $\left\{\Lambda_{i}(t)\right\}$ converge monotonically $\left(\Lambda_{i}(t) \geq E_{i}^{e x}, \forall t\right)$ to the exact energies $\left\{E_{i}^{e x}\right\}$ in the limit of infinite imaginary time,

$$
\begin{gathered}
\lim _{t \rightarrow \infty} \Lambda_{i}(t)=E_{i}^{e x}, \\
\frac{d \Lambda_{i}(t)}{d t} \leq 0, \quad \forall t .
\end{gathered}
$$

Correlation function $\mathrm{MC}$ has the important zero variance property of the energy: As the basis set approaches the exact eigenstates, the variance of the estimates of the eigenvalues approaches zero.

The importance sampled Green's function [Eq. (61)] satisfies (in a gauge covariant form)

$$
\begin{aligned}
& \partial_{t} G\left(\mathscr{R}, \mathscr{R}^{\prime} ; t\right) \\
&=-\frac{1}{2} \nabla_{\mathscr{R}} \cdot\left[-\nabla_{\mathscr{R}} G+2 G\left(\nabla_{\mathscr{R}} \ln \left(\psi_{G}\right)-i \mathbf{A}\right)\right] \\
&-\left(E_{L}^{*}(\mathscr{R})-E_{T}\right) G,
\end{aligned}
$$

where $E_{L}=\psi_{G}^{-1} \hat{\tilde{H}} \psi_{G}$, and $E_{T}$ is a trial energy, chosen to be real-valued.

All but the term involving the vector potential of this expression for the Green's function (and the fact that $E_{L}$ is complex-valued) are commonly used in diffusion MC. Trotter's theorem asserts that under weak conditions on the (linear) operators we can consider the evolution as the product of the evolution of each of the operators separately. Hence, a short-time solution to Eq. (73) is given by the product of the three Green's functions: [corrections are $\mathscr{O}\left(\tau^{2}\right)$ ]

$$
G\left(\mathscr{R}_{j}, \mathscr{R}_{j-1} ; \tau\right)=G_{d} G_{b} G_{\mathrm{A}},
$$

where

$$
\begin{aligned}
G_{d}\left(\mathscr{R}_{j}, \mathscr{R}_{j-1} ; \tau\right)= & (2 \pi \tau)^{-3 N / 2} \\
& \times e^{-\left(\mathscr{R}_{j}-\mathscr{R}_{j-1}-\tau \mathrm{F}_{Q}\left(\mathscr{R}_{j-1}\right)\right)^{2} / 2 \tau},
\end{aligned}
$$

$$
\begin{aligned}
& G_{b}\left(\mathscr{R}_{j}, \mathscr{R}_{j-1} ; \tau\right)=e^{-\tau\left[\left(\tilde{E}_{L}\left(\mathscr{R}_{j}\right)+\tilde{E}_{L}\left(\mathscr{R}_{j-1}\right)\right) / 2-E_{T}\right]}, \\
& G_{\mathbf{A}}\left(\mathscr{R}_{j}, \mathscr{R}_{j-1} ; t\right)=e^{-i\left(\mathscr{R}_{j}-\mathscr{R}_{j-1}\right) \cdot \mathbf{A}\left(\left(\mathscr{R}_{j}+\mathscr{R}_{j-1}\right) / 2\right)},
\end{aligned}
$$


$\mathbf{F}_{Q}=-\nabla \ln \psi_{G}$ is again the quantum force, and $\tilde{E}_{L}=\psi_{G}^{-1}\left(-\frac{1}{2} \nabla_{\mathscr{R}}^{2}+V+\sigma \cdot \mathbf{B}\right) \psi_{G}$ is the local energy of the guiding function without the contribution from the vector potential, which is taken into account by $G_{A}$. One can verify that this $G$ satisfies Eq. (73) for infinitesimal times $\tau$ by substituting it into the master equation Eq. (73) and performing a Taylor expansion for small $\mathscr{R}-\mathscr{R}^{\prime}$, keeping only terms of linear order in $\tau$. In this limit of short time-steps, the solution to Eq. (73) is equivalent to considering the local energy and quantum force as constant in the neighborhood of $\mathscr{R}$. The midpoint evaluation of the vector potential in $G_{\mathbf{A}}$ is necessary to obtain the correct form of the Schrödinger equation (a problem related to the Ito integral [40]).

In practice, one constructs $N_{T}$ trajectories sampled according to Eq. (74), building the matrices $\mathbf{h}$ and $\mathbf{s}$ for each trajectory and $\mathbf{H}$ and $\mathbf{S}$ for the sum over configurations. The resulting eigenvalue spectrum converges asymptotically to the exact energy at large imaginary times, but with an exponentially increasing variance. One hopes to obtain reliable convergence in the energies before the error bars grow too large. The computation of expectation values of observables other than the energy (density, dipole moments, etc.) are easy to obtain within this formulation. Additional details and a thorough discussion about the choice of gauge to reduce the statistical fluctuations can be found in [24]. In the following section, we consider application of the fixed-phase and released-phase methods to both isolated helium atoms and the $\mathrm{H}_{2}$ molecule in strong and superstrong magnetic fields.

\section{QMC Results for Two-body Systems: He and $\mathrm{H}_{2}$}

\section{NEUTRAL HELIUM}

The fixed-phase approach will enable us to remove the basis-set errors in our HF calculations for atoms in strong magnetic fields. We will also be able to gain insight into the role of electron correlation as a function of magnetic field strength. In general, the fixed-phase results presented here are an excellent tool for diagnosing problem areas in our HF calculations. Tabulated energies for the results in this section are included in Appendix A.
As we have already noted, next to hydrogen, helium is the most important element for astrophysical observation in strong fields. Helium also happens to be an excellent test for our HF calculations. Using the FPQMC method will enable us to resolve several important questions remaining from our HF analysis of magnetized helium. First, we can remove the bias coming from basis-set truncation errors. Second, we can estimate the importance of electron correlation as the field strength increases. From this information, we can establish whether or not our HF calculations provide reliable spectra. Figure 11 shows the behavior of the lowest electronic states for helium, and the difference between the FPQMC and the HF results (inset). The correlation energy $E_{C}$ is defined by

$$
E_{C}=E^{e x}-E_{\mathrm{HF}},
$$

where $E^{e x}$ is the exact energy and $E_{\mathrm{HF}}$ is the fully converged (infinite basis set) HF energy. From the inset, we can see that, as expected, correlation energy is increasing as a function of field strength. Unfortunately, we can also see that the basis-set errors in the HF calculations are also increasing as the field strength increases. Our basis-set errors prevent an accurate determination of the correlation energy. What are the implications for the computed spectrum? Let us examine one of the stationary lines of the helium spectrum; the zero field $1 s 3 d_{-1}$ to $1 s 2 p_{0}$ transition. Figure 12 shows this transition, determined using both HF and FPQMC. This particular spectral line is between two lowest-energy states of their respective symmetries (which we are able to compute in FPQMC). The basis-set difficulties become worse for the excited states of any symmetry, which is reflected by the much larger swings in the HF spectral line for the transition from the $1 s 3 p_{-1}$ state to the $1 s 2 s$ state, also shown in Figure 12 (dash-dotted line). What is needed is a form of QMC that can treat the excited states, which we have in the form of RPQMC.

Tabulated energies of neutral helium using the RPQMC method can be found in [24]; here, we summarize the main results. Figure 13 shows the energy spectrum for neutral helium, including the first three excited states of each symmetry having $M=0$ and $M=-1$. Note that the separation between states of the same symmetry grows larger as the field increases. The inset in Figure 13 shows 


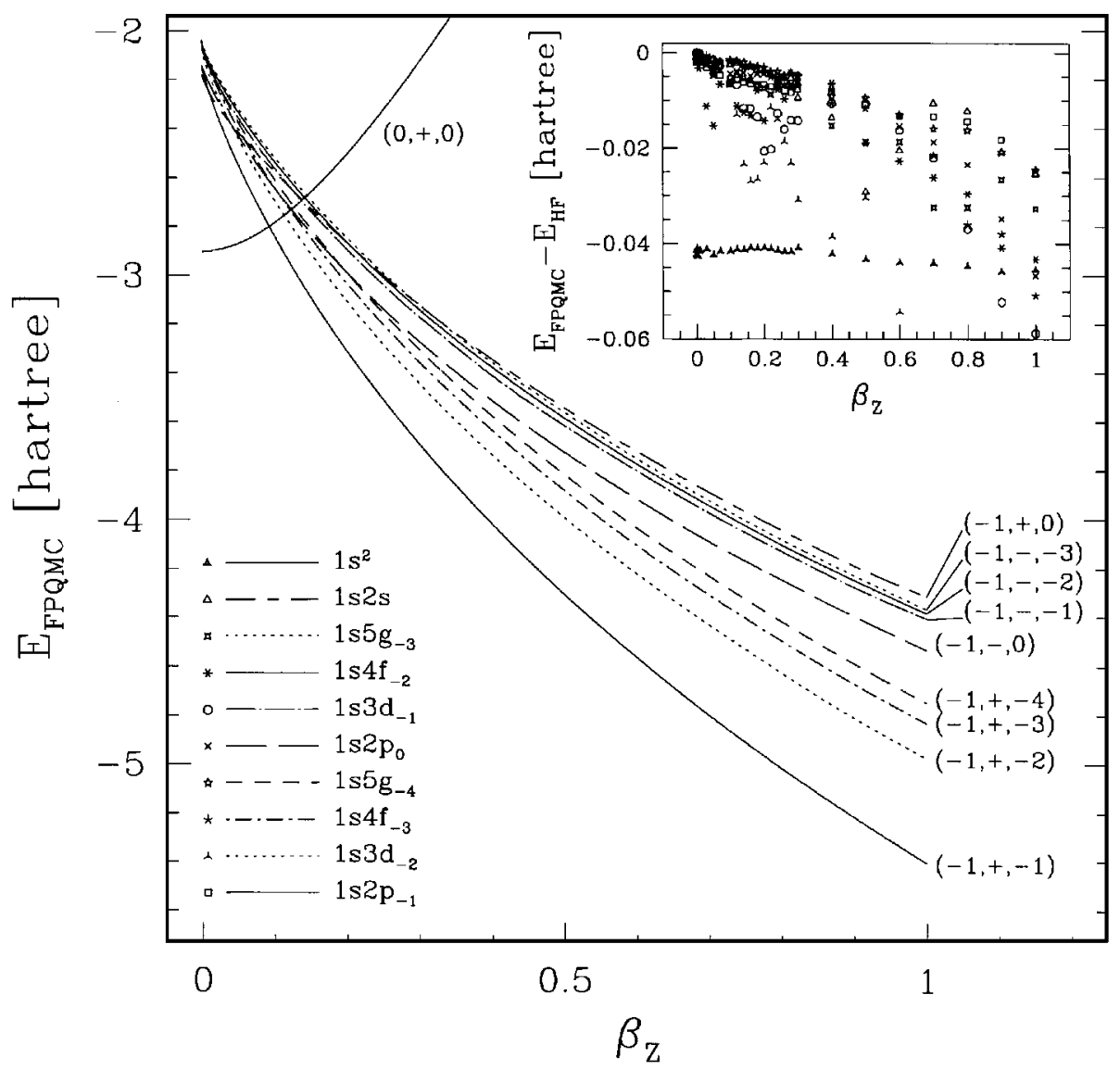

FIGURE 11. FPQMC results for neutral atomic helium. The lines are labeled by the correct quantum numbers at nonzero field, which correspond, at zero field, to the quantum numbers given in the key at the lower-left portion of the plot. The inset shows the difference from the corresponding HF results.

the difference between the energy of the second state using RPQMC and HF. We can see that the basis-set truncations error, as expected, is very much worse for the more highly excited states.

Using these RPQMC results, we can construct the spectrum of allowed transitions between the various electronic states. Figure 14 shows all of the spectral lines involving the first two excited states with $M=0,-1$. Most of the allowed lines are quite rapidly changing as a function of magnetic field. A few, however, are very nearly constant over wide ranges of field strength (this fact is made more clear by viewing the figure from the side). These "stationary" lines are crucial for matching observed spectra. A detailed matching of observed spectral features will require further RPQMC calculations (more values of $\hat{L}_{z}$ must be considered), which are already under way [41].

\section{THE $\mathrm{H}_{2}$ MOLECULE}

Recently [25, 42] there has been a debate on whether a hydrogen gas can become superfluid in the presence of a strong external magnetic field. The crucial argument supporting the existence of a superfluid phase is that due to weak interatomic interactions $[26,27]$ the system behaves as a weakly interacting Bose gas and as a consequence of macroscopic exchanges it becomes superfluid. However, as it has been shown in [3], the system turns out to be strongly interacting with a compelling tendency to forming a molecular phase before Bose-Einstein condensation takes place. The gist of the discrepancy lies in the assumption of different symmetries for the molecular ground states. What is the ground-state symmetry of a hydrogen molecule in the presence of an external 


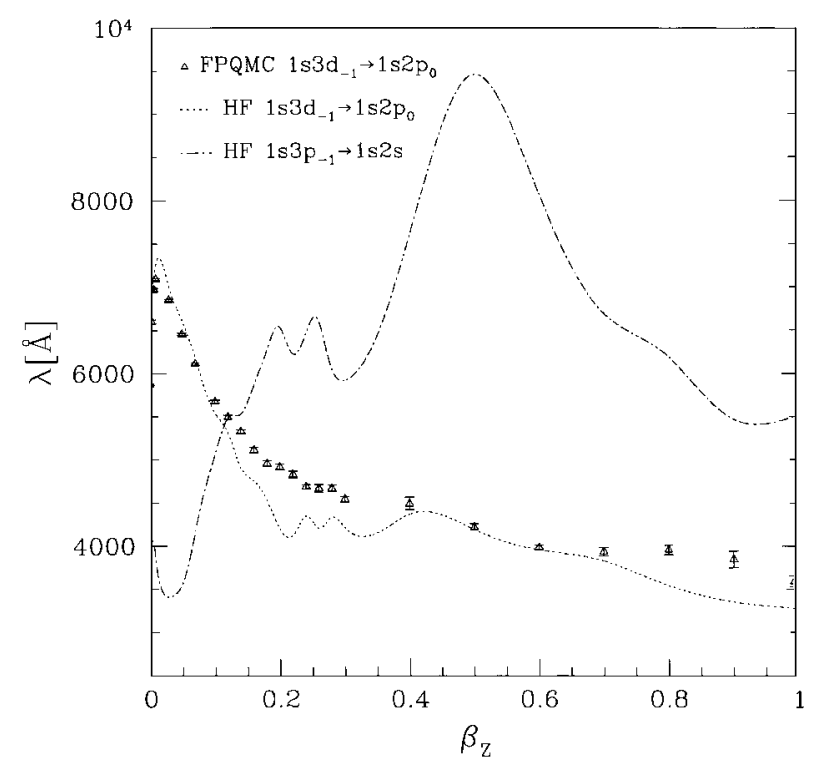

FIGURE 12. Quality of the HF helium spectrum. The points with error bars are the FPQMC wavelengths, while the dotted and dash-dotted lines are the HF wavelengths using a splined fit to the HF total energies. Note that the basis-set errors cause unpredictable deviations in the HF spectral lines.

magnetic field? This question and other related matters constitute the subject of this section.

We consider only the electron dynamics, which, in turn, depends parametrically on the nuclear space coordinates $\mathbf{R}_{j}(j=1,2)$ (with internuclear separation $R$ and axis whose center coincides with the origin of the coordinate reference frame) and the angle $\Theta$ between the internuclear and magnetic field axis. In the following, we will always consider the case $\Theta=0$, i.e., the so-called parallel configuration of the molecule.

Let us start by writing the nonreativistic Hamiltonian $\hat{\mathbb{H}}$ which governs the dynamics of our twofermion system in the Coulomb potential of two nuclei with infinite mass and charge $Z$ and in the presence of an external electromagnetic potential $A_{\mu}$ which we fix to be in the symmetric gauge. Then, in Hartree atomic units,

$$
\begin{aligned}
& \hat{\mathbb{U}}=\sum_{i=1}^{2}\left[-\frac{\nabla_{i}^{2}}{2}-\sum_{j=1}^{2} \frac{Z}{R_{i j}}+\frac{\beta^{2}}{2}\left(x_{i}^{2}+y_{i}^{2}\right)\right] \\
& +\beta\left(\hat{L}_{z}+2 \hat{S}_{z}\right)+\frac{1}{r_{12}}+\frac{Z^{2}}{R},
\end{aligned}
$$

where $\hat{L}_{z}=\hat{\ell}_{1 z}+\hat{\ell}_{2 z}$ and $\hat{S}_{z}=\hat{s}_{1 z}+\hat{s}_{2 z}$ are the $z$-component of the total electronic angular mo- mentum and spin of the system, respectively. $\mathbf{r}_{i}=$ $\left(x_{i}, y_{i}, z_{i}\right)$ represents the electron vector position, $R_{i j}=\left|\mathbf{r}_{i}-\mathbf{R}_{j}\right|$, and lengths are in units of the bohr radius $a_{0}$. Hence, we are dealing with spin- $1 / 2$ fermions of mass $m$ and charge - $|e|$ coupled, in principle, to both orbital and spin degrees of freedom (Zeeman term). Notice that, for simplicity, we have not considered spin-orbit coupling.

The eigenvalues of $\hat{S}^{2}, \hat{S}_{z}$, and parity $\hat{\Pi}(\hat{\Pi}|\mathscr{R}\rangle$ $=|-\mathscr{R}\rangle)$ are good quantum numbers. Since we will only consider the classically stable (minimumenergy) configuration, i.e., the one where magnetic field direction $(z)$ and internuclear axis coincide $(\Theta=0)$, one can also classify the electronic states according to the eigenvalues of $\hat{L}_{z}$, i.e., the generator of rotations about the magnetic field axis. Notice that had we considered the complete four-body problem [i.e., 2 protons $(q=|e|)$ and 2 electrons $(q=-|e|)$ [43], the total pseudomomentum $\mathbf{K}_{0}$ would have allowed a complete separation of the center of mass motion.

For the superstrong range of field strength, the sector of $S=0$ is irrelevant for the low-energy spectrum, and only the completely spin-polarized one, $S=1$, will be analyzed. Then, the configurational part of the wave function $\Phi(\mathscr{R})$ is antisymmetric. In the following, we will determine, using stochastic techniques, the sector of $\hat{L}_{z}$ to which the ground-state $\Phi_{0}$ belongs.

As already mentioned in previous sections, the basic difficulty in solving the stationary Schrödinger equation $\hat{\mathscr{H}} \Phi=E \Phi$ for arbitrary magnetic field strength lies in the different symmetries furnished by the Coulomb and Lorentz forces, which prevent closed-form analytic solutions. Thus, to study the spectrum of $\hat{\imath}$, we will make use of the stochastic methods already described above, mainly VMC, FPQMC, and RPQMC methods, although we will concentrate on lowest-energy states belonging to sectors of a given symmetry.

To proceed with the FPQMC approach, we reformulate the nonrelativistic quantum mechanics in terms of the modulus $|\Phi|$ and phase $\varphi$ of the scalar $N$-particle state $\Phi(\mathscr{R})=|\Phi(\mathscr{R})| \exp [i \varphi(\mathscr{R})]$. Then, the stationary Schrödinger equation is equivalent to solving two real (coupled) differential equations for $|\Phi|$ and $\varphi$, which in the present context reads

$$
\begin{aligned}
\hat{H}|\Phi(\mathscr{R})| & =\left[\hat{\mathbb{U}}_{M, M_{S}}+\sum_{i=1}^{N} \frac{\mathscr{A}_{i}^{2}(\mathscr{R})}{2}\right]|\Phi(\mathscr{R})| \\
& =E|\Phi(\mathscr{R})|
\end{aligned}
$$




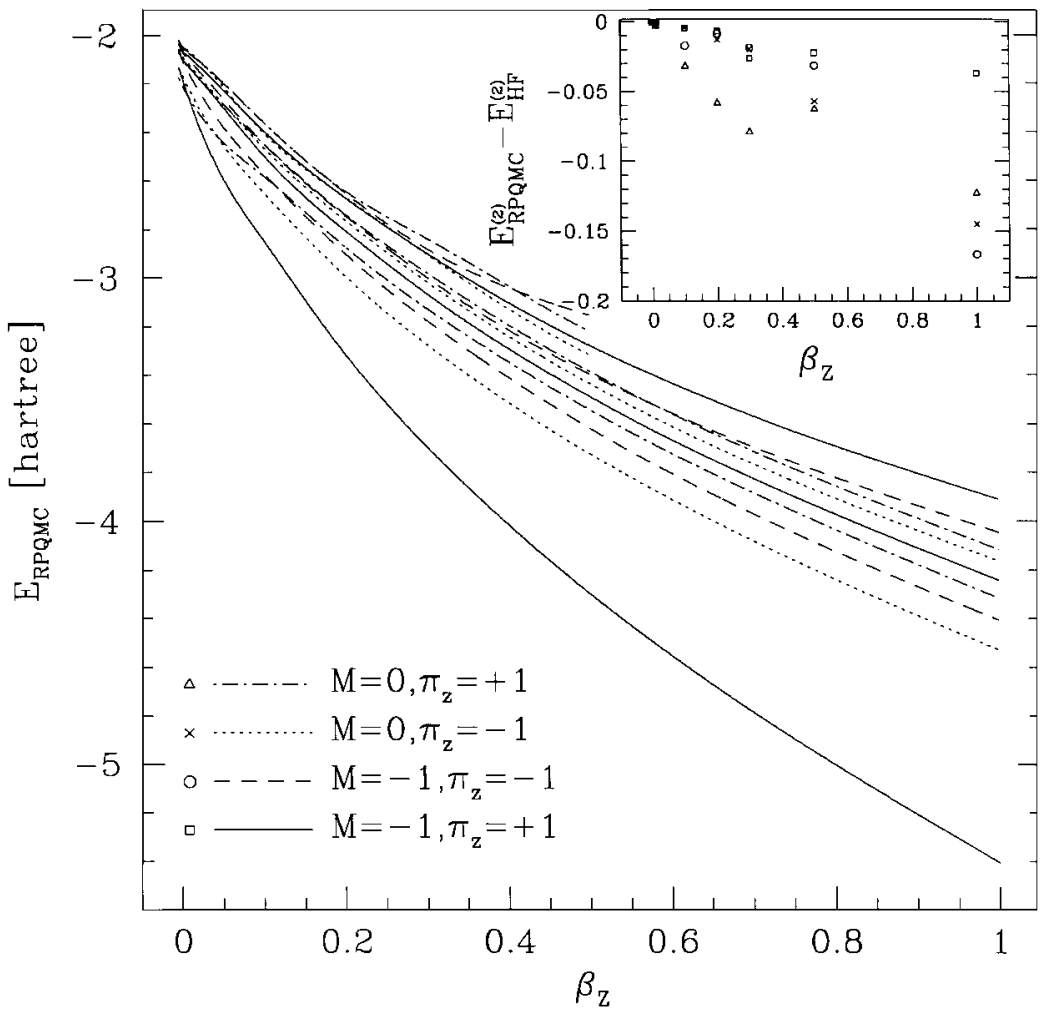

FIGURE 13. The helium energy spectrum using RPQMC. The lines are spline fits to our data. Three states were evaluated for each of the four symmetries shown. The inset shows the difference of the second-state's energy from HF.

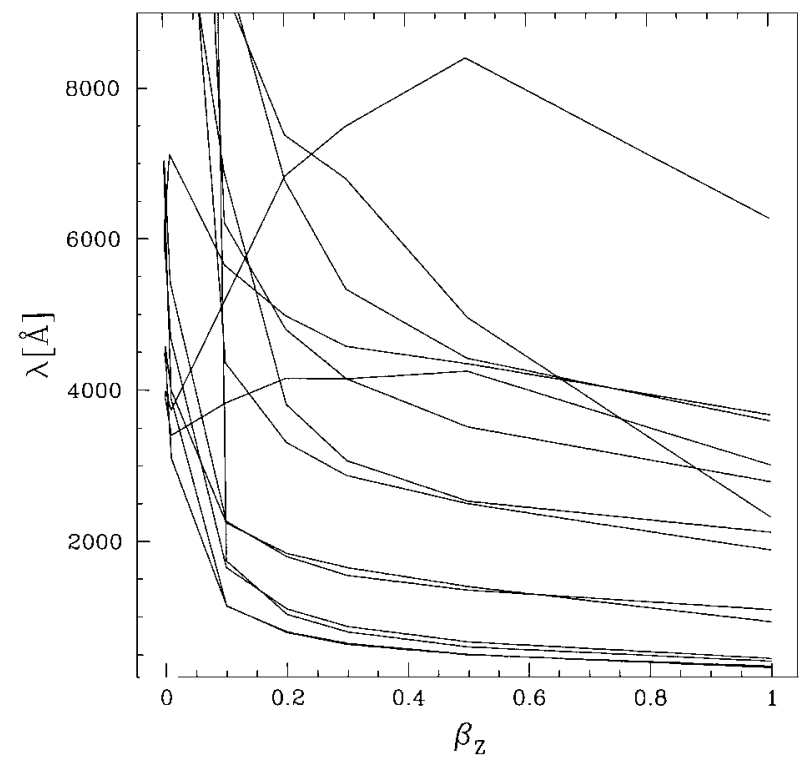

FIGURE 14. Wavelengths of allowed dipole transitions for helium.

$$
\sum_{i=1}^{N} \nabla_{i} \cdot\left[|\Phi(\mathscr{R})|^{2} \mathscr{A}_{i}(\mathscr{R})\right]=0,
$$

where $\hat{\mathbb{H}}_{M, M_{S}}$ is the Hamiltonian of Eq. (79) already projected onto the subspace with quantum numbers $M$ and $M_{S}$. Once a trial phase $\varphi_{T}$ has been chosen, we solve the eigenvalue Eq. (80) within each subspace $\left(M, M_{S}\right)$ using randomwalks.

The phase $\varphi_{T}\left(M, M_{S}\right)$ is chosen from the set of trial functions $\left\{\Phi_{T}^{n \bar{n}}\right\}$, which are going to constitute the basis functions for the RPQMC method, with

$$
\begin{aligned}
\Phi_{T}^{n \bar{n}}(\mathscr{R})= & \exp \left[\frac{r_{12}}{a_{\zeta}+\sqrt{c_{1} \rho_{12}^{2}+c_{2} z_{12}^{2}}}\right. \\
& \left.-\sum_{i j} \frac{Z R_{i j}}{1+b R_{i j}}\right] \\
& \times\left[g_{+}^{n}\left(\mathbf{r}_{1}\right) g_{-}^{\bar{n}}\left(\mathbf{r}_{2}\right)+\zeta g_{+}^{n}\left(\mathbf{r}_{2}\right) g_{-}^{\bar{n}}\left(\mathbf{r}_{1}\right)\right],
\end{aligned}
$$


whose modulus is used as an importance-function to guide the random-walk. In Eq. (82), $\zeta=1$ for $S=0$ and $\zeta=-1$ for $S=1$. The one-body oribitals are chosen as

$g_{ \pm}^{n}(\rho, \phi, z)=\rho^{|m \pm|} \exp \left[i m_{ \pm} \phi-F_{ \pm}^{n}(\rho, z)\right]$,

with

$$
F_{ \pm}^{n}=\mu \rho^{2}+\frac{\kappa_{ \pm}\left|z_{ \pm}\right|^{2}}{1+\alpha_{ \pm}\left|z_{ \pm}\right|}+\ln G_{ \pm}^{n}
$$

and

$$
G_{ \pm}^{n}=\left|z_{ \pm}\right|^{1-\delta_{n, 0}}\left[\nu_{1}+\left|z_{ \pm}\right|^{\nu_{2}}\right] \sum_{j=1}^{n}\left(1-\gamma_{j}\left|z_{ \pm}\right|\right)
$$

where $z_{ \pm}=z \pm \hat{R}$, and $c_{i}, b, \mu, \kappa_{ \pm}, \alpha_{ \pm}, \hat{R}, \nu_{i}$, and $\gamma_{i}$ are variational parameters. The full trial functions, in addition to having the product of one-body states $g_{ \pm}^{n}$, also have a Jastrow factor with electron-electron and electron-nuclear two-body correlation functions which satisfy Kato cusp conditions at the collision points $\left(a_{\zeta}=2[S=0]\right.$ or 4 $[S=1])$. The pair of indices $[n, \bar{n}]$ distinguish the different excitations of a given symmetry. For instance, $[n=0, \bar{n}=0]$ characterizes the ground states and $[n=1, \bar{n}=0]$ (or $[n=0, \bar{n}=1$ )], the first excited states of a given symmetry. Note that this set of states is going to be a good representation of the true excitations only in the strong magnetic field limit; otherwise, it is linearly independent set. It is straightforward to prove that $\Phi_{T}^{n \bar{n}}$ is an eigenstate of $\hat{L}_{z}$ with eigenvalue $M=m_{+}+$ $m_{-}$, and for $\tilde{R}=0$, it is s state of parity $(-1)^{M}$ (or $z$-parity +1 ).

We start our calculations at the VMC level in the variance minimization version [44]. To this end, we vary the free parameters in $\Phi_{T}^{n \bar{n}}$ in order to minimize the fluctuations in the local energy $\sigma^{2}=\int d \mathscr{R}\left|\Phi_{T}^{n \bar{n}}\right|^{2}\left[E_{L}(\mathscr{R})-E_{T}\right]^{2} / \int d \mathscr{R}\left|\Phi_{T}^{n \bar{n}}\right|^{2}$. This strategy provides a balanced optimization of the wave function and has a known lower bound (namely, zero). Once the trial wave function has been optimized, we use the walkers generated with a multiparticle force-bias Metropolis algorithm to compute the expectation value of the observables of interest, which for our present purposes consists only of the total energy spectrum $E_{M, M_{S}}=\left\langle\Phi_{T}^{n \bar{n}} \mid \hat{\mathbb{H}} \Phi_{T}^{n \bar{n}}\right\rangle /\left\langle\Phi_{T}^{n \bar{n}} \mid \Phi_{T}^{n \bar{n}}\right\rangle$. The results of this calculation $(Z=1)$ for $[n=0, \bar{n}=0]$ are de- picted in Figure 15, for two different values of magnetic field strength. In this figure, we show the total energies as a function of the internuclear separation $R$ for two different symmetry states, namely, $\left(M, M_{S}\right)=(0,-1)$ and $(-1,-1)$. The energy of the state $(0,-1)$ decreases monotonically as a function of increasing $R$ reaching asymptotically the limit of two isolated $\mathrm{H}$ atoms in the $1 \mathrm{~s}$ state and constitutes a repulsive state. On the other hand, the state $(-1,-1)$, which is the ground state in this superstrong regime, presents a deep minimum at the equilibrium nuclear separation $R_{e}$ with a limiting energy value which corresponds to having one $\mathrm{H}$ atom in the $1 \mathrm{~s}$ and another in the $2 p_{-1}$ states. In the $R \rightarrow \infty$ limit, our trial wave

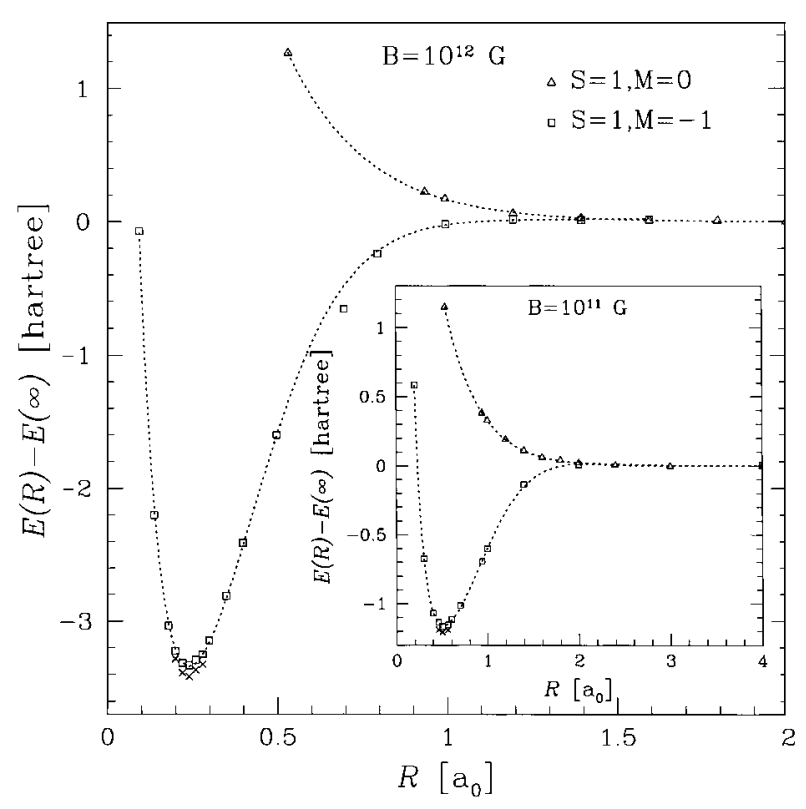

FIGURE 15. The VMC total energy of $\mathrm{H}_{2}$ as a function of the internuclear separation $R$ for the $(0,-1)$ and $(-1,-1)$ states. The symbols correspond to the MC calculations while the dotted lines are the result of a fit to a modified Morse potential. The energies are defined with respect to their values in the infinite separation limit, which are $E_{(0,-1)}(\infty)=-11.925(4)$ and $E_{(-1,-1)}(\infty)=$ $-10.234(11)$ in Hartree atomic units. These can be compared to the exact [3] atomic values (after interpolation) -11.9206 and -10.2603 , respectively. For comparison, we also show the FPQMC energy results (crosses) around the equilibrium configuration. The inset corresponds to a different magnetic field strength. In this case, $E_{(0,1)}(\infty)=-5.7197(21)$ and $E_{(-1,-1)}(\infty)=$ $-4.794(4)$, while the exact atomic values are -5.7185 and -4.7984 , respectively. 
function yields the exact energies [1] within the statistical error bar. However, for the largest magnetic fields considered, some correlation energy is missing in $E_{(-1,-1)}(\infty)$. This small energy difference is restored with our FPQMC method, which, in the above-mentioned limit, is essentially exact because the nodal surface structure of the manyfermion wave function is irrelevant.

To determine the bonding parameters, we fit the VMC data to the modified Morse potential of Hulburt and Hirschfelder [45, 46] and used this function (and not the Hellman-Feynman theorem $\left.\left\langle\Phi_{T}^{n \bar{n}}\left|\partial_{R} \hat{\mapsto}\right| \Phi_{T}^{n \bar{n}}\right\rangle=0\right)$ to compute them. Table III displays these results. As a function of increasing field strength, the molecule gets smaller and the dissociation energy increases with suggests that a low density gas of $\mathrm{H}$ atoms under such conditions has a tendency to form a strong bonded molecular phase and not a superfluid one as has been proposed [25-27, 42].

To go beyond the VMC results, we start our FPQMC computation assuming the phase $\varphi_{M, M_{S}}$ within each subspace. Recall that FPQMC provides a variational upper bound only for $[n=0, \bar{n}=0]$. We begin $\tau=0$ with an ensemble of $N_{c}=200$ configurations $\mathscr{R}_{i}\left(i=1, \ldots, N_{c}\right)$ distributed according to $P(\mathscr{R})=\left|\Phi_{T}^{n \bar{n}}\right|^{2}$, then diffuse and drift each configuration as $\mathscr{R}_{i}^{\prime}=\mathscr{R}_{i}+\tau \mathrm{F}_{Q}\left(\mathscr{R}_{i}\right)+\eta$, where $\eta$ is a normally distributed random vector with a variance of $\tau$ and branch with the local energy. The total number of configurations is then relaxes by propagation in imaginary time and stabilized when it approximates the stationary distribution $P(\mathscr{R}, \tau \rightarrow \infty) \rightarrow\left|\Phi_{T}^{n \bar{n}}(\mathscr{R}) \| \Phi_{M, M_{s}}(\mathscr{R})\right|$. In Table III, we present the FPQMC ground-state results at the equilibrium nuclear configuration $R_{e}$ and compare them to the HF calculations at Lai et al. [17] We find about 2\% lower energy. For the subspace $(0,-1)$, in the range of magnetic field strengths considered, the the FPQMC approach does not correct the VMC energy values within the statistical uncertainty (and this is also for the exact RPQMC results, see Table IV), reflecting the high quality of the trial wave function used.

Finally, let us summarize our analysis of the ground-state symmetry as a function of increasing magnetic field strength. In the weak field regime $\left(\beta \leq 10^{-3}\right)$, the ground state belongs to the subspace $(0,0){ }^{1} \Sigma_{0}$, while in the superstrong regime $\left(3 \times 10^{3}>\beta \gg 1\right)$, it belongs to $(-1,-1)^{3} \Pi_{0}$. This non-time-reversal invariant state has a strong interatomic interaction suggesting that a hydrogen gas will form a strong bonded molecular phase and not a Bose-Einstein condensate as has been suggested in $[26,27]$, whose conclusion was based on the wrong symmetry state [namely $\left.(0,-1)^{3} \Sigma_{0}\right]$. The singlet-triplet transition takes place in the intermediate field regime ( $\beta \approx 0.3$ ), as indicated in Figure 16. It seems instructive to point out that a similar symmetry transition happens in a He atom [24]. This is not surprising since a He atom is a $\mathrm{H}_{2}$ molecule with zero internuclear separation. $M$ symmetry phase transitions have been predicted, in a different context, for quantum dot He [47].

\section{Conclusions}

In our studies of the behavior of multielectron atoms and molecules, we have clearly favored a many-body approach. Our methodology has been systematic, beginning with a mean-field, singleparticle description (Hartree-Fock), then progressing to successive stochastic approaches to solving the Schrödinger equation. The first projector Monte Carlo method that we applied was the fixed-phase approach, which uses an approximate phase for the trial wave function and exactly solves the resulting bosonic equation for the modulus. This technique allowed us, within the limiting uncertainty of the quality of the trial phase, to remove the deficiencies of the single-particle orbital description. We then applied the released-phase quantum Monte Carlo method, which is able to relax the fixed-phase approximation and obtain an exact solution for the energy of a small manyfermion system. By following this path, we were able to systematically work toward an exact solution in the most illustrative way. This broad approach has enabled us to observe some striking physical changes in strongly magnetized systems.

The first observation of general character regarding the physics of finite Coulomb fermion systems in the presence of external magnetic fields is that in the small magnetic field limit regular perturbation expansions in the field strength are well defined, while that is not the case in the opposite limit where Coulomb operators represent singular perturbations and asymptotic expansions are needed. From the theoretical viewpoint, the intermediate field regime (i.e., the one where Coulomb and Lorentz forces are nearly equal importance) is the most challenging one. 


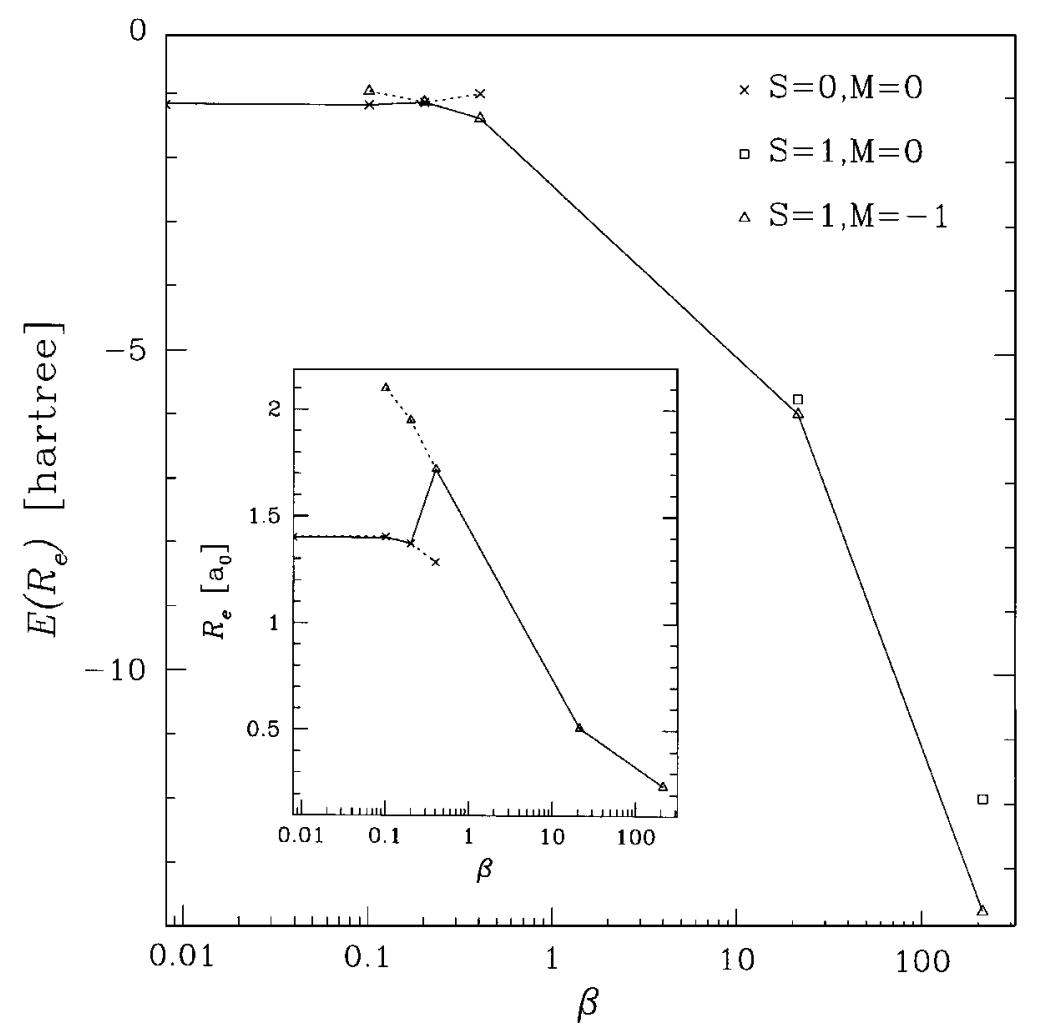

FIGURE 16. Structural ground-state properties of $\mathrm{H}_{2}$ as a function of magnetic field strength. $E\left(R_{e}\right)$ is the total energy at the equilibrium internuclear separation $R_{e}$. Notice that $R_{e}$ increases at the singlet-triplet transition. Because of the repulsive nature of the state $(0,-1){ }^{3} \Sigma_{0}$, the squares represent its energy value for $R \rightarrow \infty$. The lines are just a guide for the eye.

In general, there is an increase in the binding energy of atomic and molecular systems as the magnetic field gets larger. The increase in the binding comes from the result of strong localization of the electrons around the nuclei. In the adiabatic limit $(B \rightarrow \infty)$, the particles essentially live in quantized Landau orbits in the $(x y$-)plane perpendicular to the magnetic field $(z-)$ axis, while they feel an average Coulomb-like $1 /(|z|+\Gamma(B))$ potential in the parallel direction (with $\Gamma$ a magnetic field dependent constant). This argument is, of course, a qualitative noninteracting picture of the most relevant physical behavior.

More interesting and rich phenomena, however, are predicted when the electron-electron Coulomb repulsion is taken into account. A competition results among rotational, Coulomb, and Zeeman energies; as the field gets larger, the system tends to shrink. To minimize the Coulomb repulsion, the system prefers to raise the angular momentum and partially spin polarize, increasing in this way the average distance between electrons. We were thus able to make a series of predictions regarding quantum transitions between ground states belonging to different symmetry sectors in atomic and molecular systems.

Another interesting and general phenomena for molecules is the contraction of the internuclear bond-length $R_{e}$ as the field strength increases (see Fig. 17). Notice, however, that at a quantum transition there is, in general, a discontinuous change of $R_{e}$. It is quite relevant to ask in what way these quantum transitions will affect the ground-state properties of extended matter. However, this is at present an open question and an opportunity for future research. 


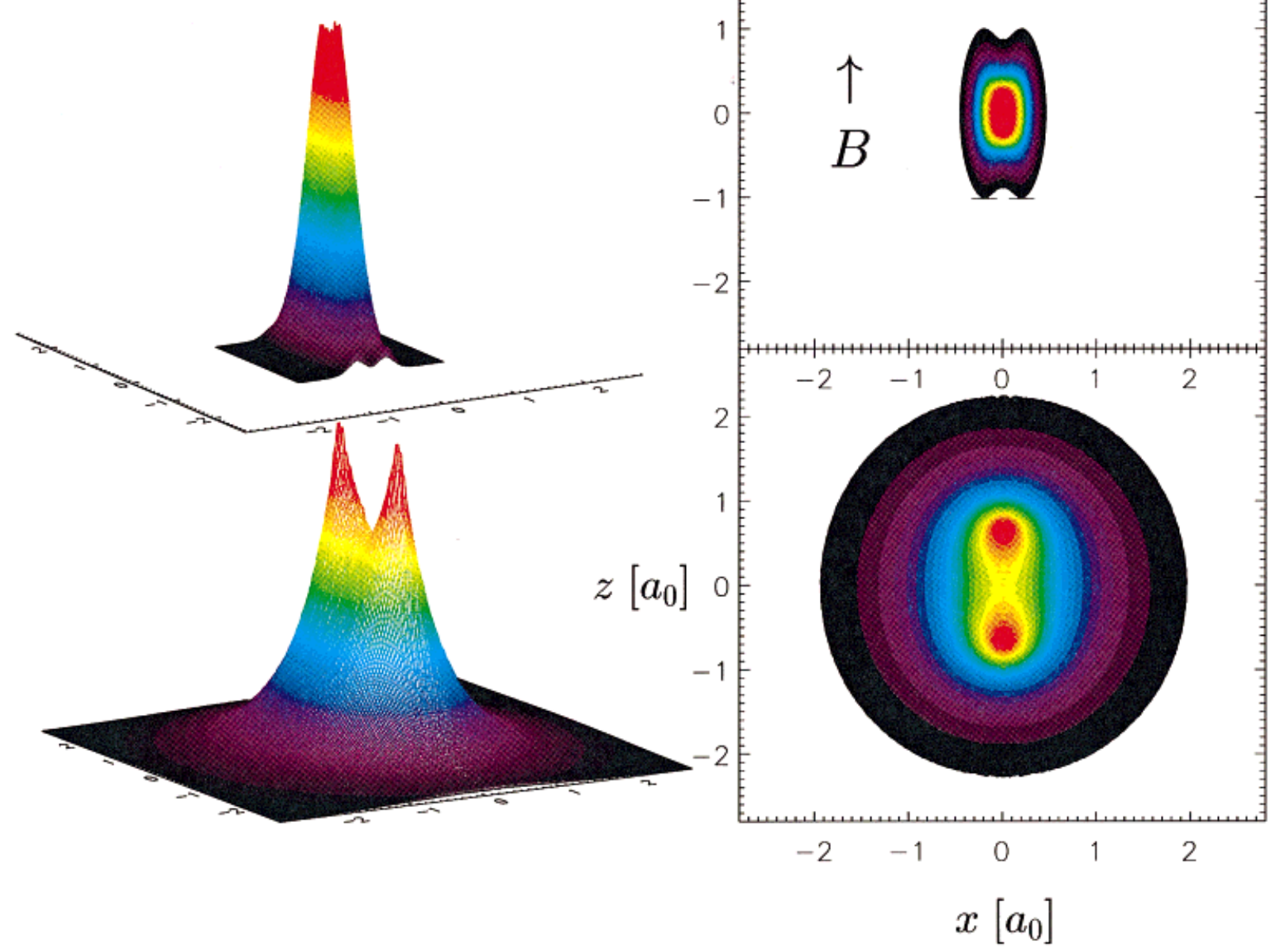

FIGURE 17. Ground state electron density plots for the $\mathrm{H}_{2}$ molecule at $B=0$ (lower panels) and $B=10^{11} \mathrm{G}$ (upper panels). Note the change in symmetry $\left({ }^{1} \Sigma_{0} \rightarrow{ }^{3} \Pi_{0}\right)$ and the contraction of the equilibrium internuclear bond-length with increasing field strength. 


\section{Appendix A: Tables of FPQMC Energies for Neutral HE}

This appendix includes tables of atomic energies from our fixed-phase QMC calculations for neutral He described in subsection Neutral Helium.

TABLE I

FPQMC energies, $E_{\mathrm{FPQMC}}$, for $\mathrm{He} M=0,-1$ states, in Hartree; numbers in parentheses are the uncertainties for each energy; zero-field quantum number are at the top of each column, along with the quantum numbers $\left(M_{S}, \pi_{z}, M\right)$.

\begin{tabular}{|c|c|c|c|c|c|}
\hline$\beta_{\mathrm{Z}}$ & $\begin{array}{c}1 s^{2} \\
(0,+, 0) \\
-E_{\mathrm{FPQMC}}\end{array}$ & $\begin{array}{c}1 s 2 s \\
(-1,+, 0) \\
-E_{\mathrm{FPQMC}}\end{array}$ & $\begin{array}{c}1 s 2 p_{0} \\
(-1,-, 0) \\
-E_{\mathrm{FPQMC}}\end{array}$ & $\begin{array}{c}1 s 2 p_{-1} \\
(-1,+,-1) \\
-E_{\mathrm{FPQMC}}\end{array}$ & $\begin{array}{c}1 s 3 d_{-1} \\
(-1,-,-1) \\
-E_{\mathrm{FPQMC}}\end{array}$ \\
\hline 0.0000 & $2.90314(52)$ & $2.17527(5)$ & $2.13318(10)$ & $2.13309(8)$ & 2.05561 (3) \\
\hline 0.0001 & 2.90396(24) & $2.17596(5)$ & $2.13391(12)$ & $2.13437(11)$ & $2.05683(1)$ \\
\hline 0.0005 & $2.90271(39)$ & $2.17915(4)$ & $2.13713(11)$ & $2.13936(10)$ & $2.06150(3)$ \\
\hline 0.0010 & $2.90320(47)$ & $2.18291(13)$ & $2.14100(11)$ & $2.14480(11)$ & $2.06711(2)$ \\
\hline 0.0030 & $2.90425(38)$ & $2.19819(6)$ & $2.15608(22)$ & $2.16768(6)$ & $2.08703(2)$ \\
\hline 0.0070 & $2.90271(29)$ & $2.22553(8)$ & $2.18509(11)$ & $2.20943(6)$ & $2.11972(3)$ \\
\hline 0.0100 & 2.90194(31) & $2.24431(23)$ & $2.20526(10)$ & $2.23843(7)$ & $2.14102(3)$ \\
\hline 0.0300 & $2.89164(32)$ & $2.33958(7)$ & $2.32248(12)$ & $2.40222(10)$ & $2.25598(6)$ \\
\hline 0.0500 & $2.87337(32)$ & $2.41255(13)$ & $2.42282(15)$ & $2.54058(9)$ & $2.35227(8)$ \\
\hline 0.0700 & $2.84465(27)$ & $2.47880(17)$ & $2.51393(14)$ & $2.66538(13)$ & $2.43939(12)$ \\
\hline 0.1000 & $2.78848(33)$ & $2.57357(12)$ & $2.63916(22)$ & $2.83541(13)$ & $2.55884(16)$ \\
\hline 0.1200 & $2.74241(34)$ & $2.63528(11)$ & $2.71526(25)$ & $2.93997(14)$ & $2.63238(18)$ \\
\hline 0.1400 & $2.69119(29)$ & $2.69573(16)$ & $2.78840(26)$ & $3.03914(17)$ & $2.70287(24)$ \\
\hline 0.1600 & 2.63436(32) & $2.75462(21)$ & $2.85831(31)$ & $3.13306(17)$ & $2.76922(42)$ \\
\hline 0.1800 & $2.57341(44)$ & $2.81194(19)$ & $2.92407(32)$ & $3.22279(17)$ & $2.83220(55)$ \\
\hline 0.2000 & $2.50822(36)$ & $2.86707(17)$ & $2.98730(38)$ & $3.30833(19)$ & $2.89464(55)$ \\
\hline 0.2200 & $2.43965(47)$ & $2.92136(23)$ & $3.04764(38)$ & $3.39101(20)$ & $2.95322(69)$ \\
\hline 0.2400 & $2.36748(47)$ & $2.97398(34)$ & $3.10680(35)$ & $3.46964(21)$ & $3.00952(37)$ \\
\hline 0.2600 & $2.29280(31)$ & $3.02474(30)$ & $3.16382(39)$ & $3.54640(22)$ & $3.06558(83)$ \\
\hline 0.2800 & $2.21476(41)$ & $3.07349(32)$ & $3.21782(35)$ & $3.61991(23)$ & $3.13014(74)$ \\
\hline 0.3000 & $2.13319(58)$ & $3.12208(35)$ & $3.27038(39)$ & $3.69166(23)$ & $3.17008(55)$ \\
\hline 0.4000 & $1.69793(46)$ & $3.34390(38)$ & $3.51253(47)$ & $4.01773(24)$ & $3.41102(162)$ \\
\hline 0.5000 & $1.21901(35)$ & $3.54353(68)$ & $3.72389(45)$ & $4.30587(27)$ & $3.61588(80)$ \\
\hline 0.6000 & $0.70766(38)$ & $3.72047(142)$ & $3.91508(56)$ & $4.56337(29)$ & $3.80064(74)$ \\
\hline 0.7000 & $0.17071(40)$ & $3.88844(77)$ & $4.08849(113)$ & $4.79854(32)$ & 3.97264(99) \\
\hline 0.8000 & $-.38570(48)$ & $4.04182(75)$ & $4.24413(122)$ & $5.01602(34)$ & $4.12888(105)$ \\
\hline 0.9000 & $-.95829(32)$ & $4.18773(215)$ & $4.39429(262)$ & $5.21577(40)$ & $4.27571(145)$ \\
\hline 1.0000 & $-1.54628(67)$ & $4.31429(208)$ & $4.53277(143)$ & $5.40452(50)$ & $4.40580(173)$ \\
\hline
\end{tabular}

TABLE II

FPQMC energies, $E_{\mathrm{FPQMC}}$, for He $M=-2,-3,-4$ states, in Hartree; numbers in parentheses are the uncertainties for each energy; zero-field quantum numbers are at the top of each column, along with the quantum numbers $\left(M_{S}, p_{z}, M\right)$.

\begin{tabular}{lccccc}
\hline & $1 s 3 d_{-2}$ & $1 s 4 f_{-2}$ & $1 s 4 f_{-3}$ & $1 s 5 g_{-3}$ & $1 s 5 g_{-4}$ \\
$\beta_{\mathrm{Z}}$ & $\begin{array}{c}1,+,-2) \\
-E_{\mathrm{FPQMC}}\end{array}$ & $\begin{array}{c}(-1,-,-2) \\
-E_{\mathrm{FPQMC}}\end{array}$ & $\begin{array}{c}(-1,+,-3) \\
-E_{\mathrm{FPQMC}}\end{array}$ & $\begin{array}{c}(-1,-,-3) \\
-E_{\mathrm{FPQMC}}\end{array}$ & $\begin{array}{c}(-1,+,-4) \\
-E_{\mathrm{FPQMC}}\end{array}$ \\
\hline 0.0000 & $2.05567(5)$ & $2.03127(1)$ & $2.03127(1)$ & $2.020002(6)$ & $2.020001(4)$ \\
0.0001 & $2.05725(2)$ & $2.03284(6)$ & $2.03324(1)$ & $2.021953(3)$ & $2.022338(4)$ \\
0.0005 & $2.06343(3)$ & $2.03878(8)$ & $2.04063(1)$ & $2.028865(4)$ & $2.030610(5)$
\end{tabular}

(Continued) 
TABLE II

(Continued)

\begin{tabular}{|c|c|c|c|c|c|}
\hline$\beta_{Z}$ & $\begin{array}{c}1 s 3 d_{-2} \\
(-1,+,-2) \\
-E_{\mathrm{FPQMC}}\end{array}$ & $\begin{array}{c}1 s 4 f_{-2} \\
(-1,-,-2) \\
-E_{\mathrm{FPQMC}}\end{array}$ & $\begin{array}{c}1 s 4 f_{-3} \\
(-1,+,-3) \\
-E_{\mathrm{FPQMC}}\end{array}$ & $\begin{array}{c}1 s 5 g_{-3} \\
(-1,-,-3) \\
-E_{\mathrm{FPQMC}}\end{array}$ & $\begin{array}{c}1 s 5 g_{-4} \\
(-1,+,-4) \\
-E_{\mathrm{FPQMC}} \\
\end{array}$ \\
\hline 0.0010 & $2.07078(3)$ & $2.04548(1)$ & $2.04887(1)$ & $2.036062(11)$ & $2.039148(6)$ \\
\hline 0.0030 & $2.09683(2)$ & $2.06710(3)$ & $2.07545(2)$ & $2.057614(12)$ & $2.064971(11)$ \\
\hline 0.0070 & $2.13922(4)$ & $2.09974(11)$ & $2.11595(2)$ & $2.08933(3)$ & $2.10327(3)$ \\
\hline 0.0100 & $2.16653(4)$ & $2.12050(6)$ & $2.14157(6)$ & $2.10950(3)$ & $2.12759(4)$ \\
\hline 0.0300 & $2.31269(17)$ & $2.23242(70)$ & $2.27883(9)$ & $2.21919(19)$ & $2.25892(16)$ \\
\hline 0.0500 & $2.43371(14)$ & $2.32706(25)$ & $2.39372(35)$ & $2.31133(29)$ & $2.36800(39)$ \\
\hline 0.0700 & $2.54193(17)$ & $2.41283(16)$ & $2.49555(18)$ & $2.39693(20)$ & $2.46740(35)$ \\
\hline 0.1000 & $2.68921(34)$ & $2.53125(22)$ & $2.63634(16)$ & $2.51328(32)$ & $2.60391(22)$ \\
\hline 0.1200 & $2.78035(43)$ & $2.60389(24)$ & $2.72273(7)$ & $2.58649(38)$ & $2.68833(29)$ \\
\hline 0.1400 & $2.86785(41)$ & $2.67408(36)$ & $2.80539(19)$ & $2.65556(26)$ & $2.76837(23)$ \\
\hline 0.1600 & $2.94915(29)$ & $2.73944(43)$ & $2.88376(21)$ & $2.72155(35)$ & $2.84453(31)$ \\
\hline 0.1800 & $3.02834(45)$ & $2.80351(35)$ & $2.95925(26)$ & $2.78514(41)$ & $2.91777(39)$ \\
\hline 0.2000 & $3.10302(94)$ & $2.86451(97)$ & $3.03128(24)$ & $2.84601(39)$ & $2.98780(44)$ \\
\hline 0.2200 & $3.17701(21)$ & $2.92333(35)$ & $3.10034(32)$ & $2.90484(51)$ & $3.05550(36)$ \\
\hline 0.2400 & $3.24655(29)$ & $2.97971(43)$ & $3.16734(36)$ & $2.93121(33)$ & $3.12058(24)$ \\
\hline 0.2600 & $3.31441(53)$ & $3.03465(52)$ & $3.23134(40)$ & $3.01625(47)$ & $3.18374(26)$ \\
\hline 0.2800 & $3.37859(45)$ & $3.08789(46)$ & $3.29436(37)$ & $3.06908(59)$ & $3.24398(28)$ \\
\hline 0.3000 & $3.44287(31)$ & $3.13945(20)$ & $3.35534(30)$ & $3.11896(60)$ & $3.30356(31)$ \\
\hline 0.4000 & $3.73472(52)$ & $3.37558(24)$ & $3.63404(39)$ & $3.35492(62)$ & $3.57513(48)$ \\
\hline 0.5000 & $3.99144(40)$ & $3.58366(49)$ & $3.88056(38)$ & $3.56063(69)$ & $3.81513(52)$ \\
\hline 0.6000 & $4.22073(57)$ & $3.77148(84)$ & $4.10418(52)$ & $3.74971(74)$ & $4.03342(51)$ \\
\hline 0.7000 & 4.42994(108) & $3.94161(72)$ & $4.30675(61)$ & $3.92104(133)$ & $4.22954(100)$ \\
\hline 0.8000 & 4.62566(109) & $4.10097(111)$ & 4.49503(83) & $4.07959(132)$ & $4.41611(36)$ \\
\hline 0.9000 & $4.80912(113)$ & $4.24582(66)$ & $4.67013(85)$ & $4.22629(113)$ & $4.58802(51)$ \\
\hline 1.0000 & $4.97560(113)$ & $4.38303(108)$ & $4.83102(97)$ & $4.36666(65)$ & $4.74774(72)$ \\
\hline
\end{tabular}

\section{Appendix B: Tabulated Results for the $\mathrm{H}_{2}$ Molecule}

TABLE III

Interatomic equilibrium separation $R_{e}$ (in units of $a_{0}$ ), vibrational frequency $\omega_{e}$ (in units of $10^{4} \mathrm{~cm}^{-1}$ ), and total ground-state energy $E_{(-1,1)}$ (in eV) for the $\mathrm{H}_{2}$ molecule.

\begin{tabular}{lccccr}
\hline$B\left[10^{12} \mathrm{G}\right]$ & $R_{e}$ & $\omega_{e}$ & $-E_{(-1,-1)}^{V M C}$ & $-E_{(-1,-1)}^{F P Q M C}$ & Lai et al. \\
\hline 0.1 & 0.51 & $2.37(1)$ & $162.4(1)$ & $163.03(5)$ & 160.3 \\
1.0 & 0.24 & $8.26(4)$ & $369.9(3)$ & $372.4(2)$ & 368.6 \\
\hline
\end{tabular}

\section{TABLE IV}

Comparison between VMC, FPQMC, and RPQMC ground-state energies of symmetry $(0,-1)$.

\begin{tabular}{lcccc}
\hline$B\left[10^{12} \mathrm{G}\right]$ & $R$ & $-E^{V M C}$ & $-E^{F P Q M C}$ & $-E^{R P Q M C}$ \\
\hline 0.1 & 1.2 & $5.5277(5)$ & $5.5412(107)$ & $5.5327(25)$ \\
0.1 & 3.0 & $5.7210(41)$ & $5.7184(3)$ & $5.7180(28)$ \\
1.0 & 2.4 & $11.9274(39)$ & $11.9222(8)$ & $11.9227(7)$ \\
\hline
\end{tabular}




\section{References}

1. For a comprehensive review, see H. Ruder, G. Wunner, H. Herold, and F. Geyer, Atoms in Strong Magnetic Fields (Springer, Berlin, 1994), and references therein.

2. L. I. Schiff and H. Snyder, Phys. Rev. 55, 59 (1939).

3. G. Ortiz, M. D. Jones, and D. M. Ceperley, Phys. Rev. A 52, 3405 (1995).

4. C. A. Mead and D. G. Truhlar, J. Chem. Phys. 70, 2284 (1979).

5. R. J. Elliott and R. Louden, J. Phys. Chem. Solids 15, 196 (1960).

6. Y. Wan, G. Ortiz, and P. Phillips, Phys. Rev. Lett. 75, 2879 (1995).

7. J. Trümper, W. Pietsch, C. Reppin, W. Voges, R. Staubeit, and E. Kendziorra, Astrophys. J. 219, L105 (1978).

8. J. Angel, Annu. Rev. Astron. Astrophys. 16, 487 (1978).

9. W. Rosner, G. Wunner, H. Herold, and H. Ruder, J. Phys. B 17, 29 (1984).

10. G. D. Schmidt, W. B. Latter, and C. B. Foltz, Astrophys. J. 350, 758 (1990).

11. G. D. Schmidt, R. G. Allen, P. S. Smith, and J. Leibert, Astrophys. J. 463, 320 (1996).

12. H. Bethe and E. E. Salpeter, Quantum Mechanics of One- and Two-Electron Systems (Springer-Verlag, Berlin, 1957).

13. R. O. Mueller, A. Rao, and L. Spruch, Phys. Rev. A 11, 789 (1975).

14. M. Vincke and D. Baye, J. Phys. B 22, 2089 (1989).

15. M. C. Miller and D. Neuhauser, Mon. Not. R. Astron. Soc. 253, 107 (1991).

16. D. Neuhauser, K. Langanke, and S. E. Koonin, Phys. Rev. A 33, 2084 (1986).

17. D. Lai, E. S. Salpeter, and S. L. Shapiro, Phys. Rev. A 45, 4832 (1992).

18. G. Thurner, H. Körbel, M. Braun, H. Herold, H. Ruder, and G. Wunner, J. Phys. B 26, 4719 (1993).

19. R. Henry, R. F. O'Connell, E. R. Smith, G. Chanmugam, and A. K. Rajagopal, Phys. Rev. D 9, 329 (1974).

20. G. L. Surmelian, R. Henry, and R. F. O'Connell, Phys. Lett. A 49, 431 (1974).

21. D. M. Larsen, Phys. Rev. B 20, 5217 (1979).

22. M. D. Jones and G. Ortiz, in Physical Phenomena at High Magnetic Fields II (World Scientific, River Edge, NJ, 1996), pp. $708-713$.
23. M. D. Jones, G. Ortiz, and D. M. Ceperley, Phys. Rev. A 54, 219 (1996).

24. M. D. Jones, G. Ortiz, and D. M. Ceperley, Phys. Rev. E (1997).

25. A. V. Korolev and M. A. Liberman, Phys. Rev. Lett. 74, 4096 (1995).

26. A. V. Korolev and M. A. Liberman, Phys. Rev. Lett. 72, 270 (1994).

27. A. V. Korolev and M. A. Liberman, Phys. Rev. B 47, 14318 (1993).

28. C. Roothaan, Rev. Mod. Phys. 23, 69 (1951).

29. R. McWeeney, Methods of Molecular Quantum Mechanics (Academic Press, New York, 1992).

30. M. F. Guest and V. R. Saunders, Mol. Phys. 28, 819 (1974).

31. T. Kato, Commun, Pure Appl. Math. 10, 151 (1957).

32. R. N. Hill, Phys. Rev. Lett. 38, 643 (1977).

33. J. E. Avron, I. W. Herbst, and B. Simon, Commun. Math. Phys. 79, 529 (1981).

34. E. R. Davidson and B. Feller, Chem. Rev. 86, 681 (1986).

35. G. Ortiz, D. M. Ceperley, and R. M. Martin, Phys. Rev. Lett. 71, 2777 (1993).

36. G. Ortiz and D. M. Ceperley, Phys. Rev. Lett. 75, 4642 (1995).

37. M. H. Kalos, D. Levesque, and D. Verlet, Phys. Rev. A 9, 2178 (1974).

38. D. M. Ceperley and B. Bernu, J. Chem. Phys. 89, 6316 (1988).

39. J. K. L. MacDonald, Phys. Rev. 43, 830 (1933).

40. L. S. Schulman, Techniques and Applications of Path Integration (Wiley, New York, 1981).

41. M. D. Jones, G. Ortiz, and D. M. Ceperley, unpublished.

42. D. Lai, Phys. Rev. Lett. 74, 4095 (1995).

43. M. D. Jones, G. Ortiz, and D. M. Ceperley, unpublished.

44. C. J. Umrigar, K. G. Wilson, and J. W. Wilkins, Phys. Rev. Lett. 60, 1719 (1988).

45. H. M. Hulburt and J. O. Hirschfelder, J. Chem. Phys. 9, 61 (1941).

46. H. M. Hulburt and J. O. Hirschfelder, J. Chem. Phys. 35, 1901 (1961).

47. M. Wagner, U. Merkt, and A. V. Chaplik, Phys. Rev. B 45, 1951 (1992). 